\title{
Mathematical modeling explains differential SARS CoV-2 kinetics in lung and nasal passages in remdesivir treated rhesus macaques
}

Ashish Goyal ${ }^{1}$, Elizabeth R. Duke ${ }^{1,2}$, E. Fabian Cardozo-Ojeda, ${ }^{1} \uparrow$, Joshua T. Schiffer ${ }^{1,2,3^{*}} \uparrow$

${ }^{1}$ Vaccine and Infectious Diseases Division, Fred Hutchinson Cancer Research Center

${ }^{2}$ Department of Medicine, University of Washington, Seattle

${ }^{3}$ Clinical Research Division, Fred Hutchinson Cancer Research Center

$\dagger$ These authors contributed equally to the work.

One Sentence Summary: We developed a mathematical model to explain why remdesivir has a greater antiviral effect on SARS CoV-2 in lung versus nasal passages in rhesus macaques. 


\section{$1 \quad$ Abstract}

2

3 Remdesivir was recently demonstrated to decrease recovery time in hospitalized patients with SARS-

$4 \quad \mathrm{CoV}-2$ infection. In rhesus macaques, early initiation of remdesivir therapy prevented pneumonia and

5 lowered viral loads in the lung, but viral loads increased in the nasal passages five days after therapy. We

6 developed mathematical models to explain these results. We identified that 1) drug potency is slightly

7 higher in nasal passages than in lungs, 2) viral load decrease in lungs relative to nasal passages during

8 therapy because of infection-dependent generation of refractory cells in the lung, 3) incomplete drug

9 potency in the lung that decreases viral loads even slightly may allow substantially less lung damage, and

10 4) increases in nasal viral load may occur due to a slight blunting of peak viral load and subsequent

11 decrease of the intensity of the innate immune response, as well as a lack of refractory cells. We also

12 hypothesize that direct inoculation of the trachea in rhesus macaques may not recapitulate natural

13 infection as lung damage occurs more abruptly in this model than in human infection. We demonstrate

14 with sensitivity analysis that a drug with higher potency could completely suppress viral replication and

15 lower viral loads abruptly in the nasal passages as well as the lung. 


\section{Introduction}

17

There is a desperate need for treatments for SARS CoV-2, the virus which causes COVID-19 disease. ${ }^{1}$ One unmet need for of antiviral therapy development is identification of virologic surrogates for clinically meaningful endpoints such as death or need for hospitalization. In the case of SARS-CoV-2infected people viral load can be routinely measured in nasal samples or saliva. ${ }^{2}$ However, the primary site of disease is lung tissue. Therefore, bronchoalveolar lavage (BAL) of the lungs would be an ideal sample. However, BAL is usually not necessary for diagnosis, represents an infection risk to medical personnel and is rarely performed in the care of COVID-19 patients. When BAL does occur, it is often late during disease in critically ill patients rather than at early clinical presentation. Thus, the natural kinetics of SARS CoV-2 in lungs are unknown in humans.

In humans, a double-blind, randomized, placebo-controlled trial showed that the nucleoside analog remdesivir limited the duration of illness and approached statistical significance for reduction in mortality when given later in disease ${ }^{3}$. In a separate study with an overall later time of treatment initiation, remdesivir had no effect on viral load or clinical outcome. ${ }^{4}$ A recent pre-print demonstrated that remdesivir was highly effective when initiated 12 hours after infection in rhesus macaques. ${ }^{5}$ In this context, remdesivir prevented pneumonia and limited extent of clinical illness. While there was an effect on viral shedding in serial BAL specimens, viral load in the nasal passage was unchanged relative to animals treated with a vehicle during the first several days of infection and higher starting at day 5. A critical question is whether nasal viral loads are potentially useful as a surrogate for the extent of lung disease during COVID-19 infection.

Here, we develop mathematical models that recapitulate viral load trajectories in both anatomic compartments of the infected rhesus macaques. The models explain these differences according to different underlying viral kinetics off therapy in both compartments as well as differential drug potency in nasal passage versus lung. 


\section{Results}

\section{Discrepant SARS CoV-2 viral loads in lungs and nasal passages in response to remdesivir treatment in}

rhesus macaques. In a recent pre-print article, 12 rhesus macaques were infected with $2.6 \times 10^{6}$ TCID50 of SARS-CoV-2 strain nCoV-WA1-2020 via intranasal, oral, ocular and intratracheal routes and then treated with either placebo or IV remdesivir $(10 \mathrm{mg} / \mathrm{kg}$ loading dose followed by 5 days of $5 \mathrm{mg} / \mathrm{kg}$ ) starting at 12 hours post infection. ${ }^{5}$ Overall treatment resulted in reduced severity of clinical illness, less pronounced infiltrates on chest radiograph, lower viral load by nucleic acid and viral titer measurement in bronchoalveolar lavage fluid on days 1, 3 and 7, and decreased volume of lung lesions, lung weight and inflammation on histologic post-mortem exam. ${ }^{5}$

We re-examined viral loads in BAL and nasal specimens, and noted that at days 1, 3 and 7 postinfection BAL viral loads were lower in the remdesivir arm relative to the vehicle arm by approximately a single order of magnitude (Fig 1a). Similar results were observed when viral load was measured using viral culture..$^{5}$ In nasal specimens, there was no observed difference in viral loads at days 1, 2, 3 and 4; on day 6 , there was a trend towards higher viral loads in the remdesivir treated arm; on day 5 and 7, nasal viral loads were statistically higher in the treated arm relative to vehicle (Fig. 1b).

When nasal viral loads were compared longitudinally to BAL viral loads in vehicle treated animals, viral loads were generally higher in BAL than in the nasal passages at days 1, 3 and 7 (Fig 1c); in the remdesivir-treated animals, viral loads were equivalent on days 1 and 3, but higher in nasal passages than BAL at day 7 (Fig 1d). Overall, these results suggest that remdesivir lowered viral load in the lung but appeared to have the opposite effect in nasal passages of rhesus macaques at late timepoints.

Dual compartment PK / PD model of remdesivir. To explain the differential observations in lung and nasal passages of remdesivir-treated animals, we developed a mathematical model to capture drug pharmacokinetic (PK) and pharmacodynamics (PD), as well as viral and immune dynamics. The PK model is represented in Fig $\mathbf{2}$ with equations listed in the Methods. It captures the steps following 
intravenous injection of remdesivir (GS-5734), including conversion to an alanine metabolite (GS704277) and then to the parent nucleoside GS-441524 (Nuc), the necessary phosphorylation of this molecule to achieve its active triphosphate form (NTP) as well as the distribution of these metabolites from plasma to tissue.

For single-dose PK, we fit the model to data from healthy rhesus macaques in which various intermediate metabolites were measured over time following a single injection of $10 \mathrm{mg} / \mathrm{kg}$, including the levels of NTP is PBMCs. ${ }^{6,7}$ We also simultaneously fit the model to multi-dose drug and metabolite trough levels from the infected rhesus macaques $(10 \mathrm{mg} / \mathrm{kg}$ at day 0.5 and thereafter, $5 \mathrm{mg} / \mathrm{kg}$ daily at days 2 till 6 post-infection), including a day 7 level of the Nuc in tissue at the time of necropsy on day 7 in Fig 3b. ${ }^{5}$ RDV PK parameters are listed in Table 1. The PD models assumes an $\mathrm{EC}_{50}$ or concentration of the active metabolite (NTP), at which replication is inhibited by $50 \%$.

The model is able to recapitulate the levels of remdesivir and its metabolites in healthy (Fig 3a) and infected rhesus macaques (Fig 3b).

Lung and nasal mathematical model of SARS Co-V-2 in rhesus macaques. We developed a model of viral replication in the nasal passage and lungs that includes multiple mechanisms that may occur following infection in lungs and the nasal passages (Fig 4a). This model is an adaptation of our previous model of human COVID-19 infection and includes a density-dependent death of infected cells as a proxy for an intensifying innate response to a higher burden of infection, proliferation of susceptible epithelial cells, the conversion of susceptible and/or infected cells to an infection refractory state and the movement of the virus between nasal passages and the lung. ${ }^{8}$ We fit different versions of this model to the viral loads in nasal passages and lung from 6 infected, remdesivir-treated animals, ${ }^{5}$ and 14 infected and untreated animals, including the 6 vehicle animals in the remdesivir protocol (RM7-12), ${ }^{5}$ and 8 other animals infected using the same protocol (4 of which had extended nasal viral load measures through 21 days after infection). ${ }^{9}$ Each model explored was a version of the full model in Fig $\mathbf{4 a}$ with individual components removed (Methods) 
Using model selection theory, we found that the model with minimal complexity necessary to

Model fit to viral load data from untreated rhesus macaques. The best model fit recapitulated the frequently observed trend of higher viral loads in BAL at late timepoints relative to the nasal passage in untreated macaques (Fig 5a). The model also closely captured the viral dynamics in BAL from untreated animals and mostly captured nasal viral loads as well, though outlier datapoints compromised model fit somewhat in several of the animals.

Infected cell death rates were generally higher while viral replication rates were uniformly lower in the nasal passages relative to lungs in the untreated animals (Table 4). The density-dependent exponent had a similar value in both compartments $(k=0.09)$, was similar to that predicted in humans, ${ }^{8}$ and led to a 2-2.5-fold increase in the overall death rate of infected cells at peak viral load.

\section{Model fit to viral load data from remdesivir treated rhesus macaques. The $\mathrm{PK} / \mathrm{PD}$ model (the} combination of the PK model output and the viral dynamics model shown in Fig 4b) recapitulated the observed trend of lower viral loads in BAL at late timepoints relative to the nasal passages (Fig $\mathbf{5 b}$ ). The model also captured BAL viral loads on treatment accurately while reproducing nasal viral loads as well, though outlier datapoints again compromised model fit somewhat in several of the animals. The waning of drug levels and potency after dosing is evident in simulated tracings that exhibit decelerating viral decay rates after each dose.

Increased remdesivir antiviral potency in nasal versus lung cells. The degree to which remdesivir suppressed viral replication varied somewhat across animals. Over the course of the treatment (from day 
0.5 to day 7), the mean efficacy of the RDV treatment in nasal mucosa was estimated to be $88.4 \%, 91.4 \%$, $86.9 \%, 81.0 \%, 86.2 \%$ and $83.5 \%$ in RM1, RM2, RM3, RM4, RM5 and RM6, respectively. Similarly, over the course of the treatment (from day 0 to day 7), the mean efficacy of the RDV treatment in lung was estimated to be $76.3 \%, 75.0 \%, 80.4 \%, 69.8 \%, 77.4 \%$ and $75.5 \%$ in RM1, RM2, RM3, RM4, RM5 and RM6, respectively (Fig 6). Brief reductions in RDV drug concentrations between doses related to lower active metabolite levels in cells were associated with viral re-expansion after each dose (Fig $\mathbf{5 b})$.

The antiviral potency of remdesivir was estimated using "in vivo" $\mathrm{EC}_{50}$. Whereas "in vitro" $\mathrm{IC}_{50}$ estimate the antiviral concentration needed to inhibit $50 \%$ of viral replication based on in vitro experiments, we estimate $\mathrm{EC}_{50}$ based on viral loads measured in vivo in animal or human experiments. ${ }^{10}$ Estimates for in ivio $\mathrm{EC}_{50}$ were roughly 2-fold higher (2-fold lower potency) in the lung relative to the nasal passages (Table 4). Variability in nasal viral load peak and contemporaneous viral loads between treated animals generally related to difference in in vivo $\mathrm{EC}_{50}$ rather than viral replication rate. $\mathrm{RM} 2$ had complex kinetics with low peak viral load followed by viral rebound (which was not captured by the model and may represent a drug resistant variant $)^{11}$ and was found to have the lowest $\mathrm{EC}_{50}$. One animal with accelerated viral elimination in the lung (RM5) was found to have a higher infected cell death rate in lung but similar $\mathrm{EC}_{50}$ relative to the other 5 treated animals (Table 4).

\section{Lack of viral rebound in the lung is explained by infection dependent generation of refractory cells.}

We next performed counterfactual simulations in which the six treated animals were assumed to have not received treatment $\left(\epsilon_{U}=0\right.$ and $\left.\epsilon_{\mathrm{L}}=0\right)$. The viral load trajectories in these simulations (Fig. 7) appear similar to those in untreated animals with BAL viral loads often exceeding nasal viral loads at later timepoints (Fig 5a). Comparisons of the counterfactual viral load tracings to the treated animals suggests that a majority of viral load decrease in lungs is achieved following the first dose and is then carried forward throughout the duration of therapy with unchanged decay slopes. On the other hand, in nasal passages, viral load is decreased initially during therapy but then stabilizes or even increases, leading to higher viral loads than counterfactual projections (Fig 7). 
In the nasal cavity, somewhere between day 2 and 6 of therapy, the tracings cross and viral loads

of the treated animals are predicted to exceed the counterfactual simulations of the same animals off

147 therapy (Fig. 7). The model projects that early treatment reduces viral load, thereby decreasing new early

148 infection and preventing depletion of susceptible cells in the nasal passages (Fig 8). Even without

149 assuming susceptible cell proliferation, there is an adequate population of these cells to establish a steady

150 state of viral replication (Fig 8). In the lung where remdesivir is less potent and initially susceptible cells

151 can become refractory to infection, treatment leads to a slower depletion of susceptible cells. These cells

152 are nevertheless depleted in a linear fashion as they convert to a refractory state (Fig 8). Inclusion of a

153 refractory cell compartment is therefore necessary to allow linear elimination of virus from serial BAL

154 samples.

155

Decreased cell death in the lungs of remdesivir treated animals. As an informal assessment of lung

157 damage, we longitudinally assessed cell death over time in our counterfactual simulations. In each case,

158 therapy decreased the degree of peak cell death by at least 33\% (Fig. 9). While lung damage is multi-

159 factorial during COVID-19, this finding is qualitatively compatible with the observation that early

160 remdesivir spared these 6 animals from severe clinical disease and abnormal lung histopathology.

Projected nasal and lung viral load trajectories at higher drug potency. Next, we performed sensitivity

163 analyses in which we assumed a more potent antiviral effect, which could arise either from different

164 dosing of remdesivir or a drug with a more potent drug. In nasal passages (Fig 10a) and in lungs (Fig

165 10b), the impact of the first dose is more profound with higher potency leading to a more abrupt decline

166 in viral load.

We estimate that minimum drug efficacies of $99.99 \%$ and $99.5 \%$ would be required to eliminate

168 virus from nasal passages and lungs within 5 days for a drug that is given 12 hours after infection. The

169 need for such high potency reflects the lack of a concurrent immune response at this early stage of

170 infection. 
172 Projected impact of later therapy in nasal and lung viral load kinetics. We previously predicted in

173 modeling of human infection that antiviral treatment with moderate potency would not clear viral

174 infection in the nasal passage (or sputum) if dosed prior to the peak viral load but would clear infection if

175 dose several days later. ${ }^{8}$ Our simulations of the rhesus macaque data arrive at a similar conclusion in the

176 nasal passage, that, paradoxically, later treatment with a moderate potency drug results in lower viral

177 loads, whereas treatment started before peak results in increased late viral loads (Fig 11a). In contrast, in

178 the lungs, later treatment at days 2 or 4 leads to a subsequent viral load trajectory similar to that of the

179 earlier treated animals during the later stages of infection (Fig 11b).

180 We estimate that minimum drug efficacies of $50 \%$ and $99.5 \%$ would be required to eliminate

181 virus from nasal passages and lungs within 5 days for a drug that is given 4 days after infection. This

182 result is due to the higher remaining viral load in the lungs of animals during the first untreated 5 days of

183 infection. 


\section{Discussion}

Viral load is a valid surrogate endpoint for treatment efficacy of several viruses including HIV, hepatitis B, hepatitis $\mathrm{C}$ and cytomegalovirus. ${ }^{12-16}$ It is plausible that SARS CoV-2 lung viral load is also

187 predictive of disease severity in humans. Viral loads from swabs of infected tissue provide an

188 approximation of the number of infected cells at a given point in time, and therefore the surface area of

189 infected tissue. ${ }^{17,18}$ Unfortunately, it is less certain whether viral load measurements can be leveraged for

190 SARS CoV-2 treatment response in humans because BAL is required to measure lung viral loads but

191 these are never performed longitudinally in infected people as part of routine clinical care. Experience

192 from other respiratory viruses suggests that viral load measures in the upper airway by nasal swab or

193 saliva may or may not be representative of those in the lung. ${ }^{19}$

Here we apply mathematical models to remdesivir treatment data in rhesus macaques in which

both lung and nasal viral load were measured. We identify that the relationship between lung and nasal

viral load in the context of antiviral treatment is complex and dependent on the potency and timing of more potent therapy with a lower in vivo $\mathrm{EC}_{50}$, then nasal viral loads are predicted to decrease in a linear fashion, in lock step with lung viral loads, immediately after starting treatment. Therefore, nasal viral loads in humans, measured either by duration of shedding or viral decay slope, may be a viable surrogate endpoint for lung viral load and downstream lung damage, but only in the context of a highly potent

The experimental results highlight inherent strengths and limitations of the rhesus macaque model. Nasal passage viral kinetics and histologic lung damage appear similar between humans and 
a similar parameter set explains nasal viral loads in humans and rhesus macaques during the first week of

211 infection, ${ }^{8}$ (though the acquired immune response is not modeled in the macaques because we do not

212 observe complete viral elimination within the experimental timeframe). Similarly, our modeling of human

213 data led to the prediction that a semi-potent treatment given extremely early infection might allow higher

214 late nasal viral loads, ${ }^{8}$ which was also observed in the rhesus macaque experiments described herein.

215 On the other hand, in rhesus macaques, extensive lung damage and clinic illness is observed

216 within two days of infection, which is not in keeping with severe illness in humans which emerges at least

217 a week after the initial phase of illness. ${ }^{5,21}$ We hypothesize that direct intratracheal inoculation of

218 macaques with a high viral titer results in more immediate infection of lung. In humans, a more common

219 pattern is for respiratory viruses to start replicating in the upper airway and then transmit to the lungs in a

220 second stage of infection. ${ }^{22}$ An alternative, and not mutually exclusive explanation is that the degree of

221 viral replication in the lung can also be established extremely early in humans, but that the more extensive

222 immune-mediated damage which may be correlated with the extent of early viral replication, occurs 1-2

223 weeks later. Had the rhesus macaques with the highest lung viral loads been followed for a longer period

224 of time, it is possible that a more severe pneumonia would have developed at later timepoints.

A counterintuitive result predicted by our model is that remdesivir is slightly more potent in the compartments). Nevertheless, SARS CoV-2 is not cleared in nasal passages as effectively as in the lungs while on treatment, because the effectiveness of antiviral therapies is never independent of the concurrent

229 intensity of the immune response to infection. ${ }^{10,23}$ We previously predicted that a more potent therapy is

230 needed after 2 days of SARS CoV-2 infection relative to $>5$ days after infection because there is little

231 innate immune pressure against the virus during its early expansion phase. ${ }^{8}$ As a result, despite a slight

232 blunting of initial viral loads, virus will rebound or stabilize and end up at a higher viral level in the nose

233 than in the absence of treatment. 
236 proxy for an intensifying innate response to a higher burden of infection. However, our model also

237 suggests that ongoing infection drives a percentage of lung cells to become temporarily refractory to

238 infection. Inclusion of this assumption is required to recapitulate lung viral load data and to explain the

239 observation that lung damage is severely blunted in animals receiving treatment. This assumption is

240 supported by modeling of influenza infection. ${ }^{24}$

241 There are several limitations of our approach. First, our approximation of lung damage is

242 relatively coarse based on the complexity of this post-viral inflammatory process which may be mediated

243 by factors other than number of infected cells. This is therefore a qualitative target of our modeling.

244 Second, our fits to nasal viral load are imperfect which may be due to imprecision in viral load

245 measurements as well as missed components within the model. In the case of RM2, there is substantial

246 viral rebound that may be due to incomplete innate responses to the first pulse of infection, or to de novo

247 drug resistance. Third, we only model early infection and therefore neglect the critical impact of the late

248 acquired immune response..$^{25-27}$

249 In conclusion, we demonstrate that in rhesus macaques, the non-linear forces governing SARS

$250 \mathrm{CoV}-2$ viral load trajectories in the lung and nasal passages differ substantially in the presence of a

251 partially effective antiviral therapy. To the extent that the rhesus macaque model approximates human

252 infection, nasal viral load remains a promising surrogate endpoint marker, but perhaps only in the context

253 of a highly potent antiviral therapy. 


\section{Methods.}

Experimental data. We analyzed viral load observations from nasal passages and BAL, and remdesivir and its metabolites plasma concentrations from 12 SARS-CoV-2-infected rhesus macaques in which 6 were treated with remdesivir and 6 received a vehicle control. ${ }^{5}$ Remdesivir was infused 12 hours after infection at a dose $10 \mathrm{mg} / \mathrm{kg}$ with subsequent daily doses of $5 \mathrm{mg} / \mathrm{kg}$ for 6 day., We also added viral loads from nasal passages and BAL from 8 additional untreated animals from Muster et al. ${ }^{721}$ In both studies, rhesus macaques were infected with $2.6 \times 10^{6}$ TCID50 of SARS-CoV-2 strain. Details about the infection and treatment protocol can be found in these two pre-prints.

We also analyzed more frequently sampled observations of remdesivir and its metabolites averaged from three healthy animals after a single IV infusion of $10 \mathrm{mg} / \mathrm{kg}$ of remdesivir. ${ }^{28}$

Remdesivir pharmakinetics model. We used a compartmental and metabolism pharmacokinetics (PK)

$272 k_{12}$ and $k_{23}$. Drug distribution to other tissues and back to plasma are described by parameters $k_{1 \mathrm{~T}}, k_{1 \mathrm{e}}, k_{2 \mathrm{~T}}$,

$273 k_{2 \mathrm{e}}, k_{3 \mathrm{~T}}$ and $k_{3 \mathrm{e}}$. We assumed that in other tissues the active triphosphate metabolite $\left(A_{4 \mathrm{~T}}\right)$ is metabolized

274 from the parent nucleoside at rate $k_{34}$. We finally assumed all metabolites have linear clearance with rates

$275 k_{\mathrm{c} 1}, k_{\mathrm{c} 2}, k_{\mathrm{c} 3}$, and $k_{\mathrm{c} 4}$. These assumptions are captured by the differential equations below:

\section{$276 \quad$ Plasma compartments:}

277

$$
\frac{d A_{1}}{d t}=-k_{c 1} A_{1}-k_{12} A_{1}-k_{1 T} A_{1}+k_{1 e} A_{1 T}
$$

(Prodrug GS-5734)

278

$$
\frac{d A_{2}}{d t}=-k_{c 2} A_{2}+k_{12} A_{1}-k_{23} A_{2}-k_{2 T} A_{2}+k_{2 e} A_{2 T}
$$

(Alanine metabolite GS-704277) 


$$
\frac{d A_{3}}{d t}=-k_{c 3} A_{3}+k_{23} A_{2}-k_{3 T} A_{3}+k_{3 e} A_{3 T}
$$

(Nucleoside GS-441524)
Other tissue Compartments:

(Prodrug GS-5734)

(Alanine metabolite GS-704277)

(Nucleoside GS-441524)

(Active triphosphate metabolite)

$\frac{d A_{4 T}}{d t}=k_{34} A_{3 T}-k_{c T 4} A_{4 T}$

We fixed the half-life of prodrug GS-5734 in blood $\left(\ln 2 / k_{C 1}\right)$ to 1 hour, the half-life of alanine metabolite (GS-704277) in blood ( $\left.\ln 2 / k_{C 2}\right)$ to 24 hours, ${ }^{28}$ and the half-life of the active triphosphate metabolite ( $\left.\ln 2 / k_{c T 4}\right)$ to 24 hours. ${ }^{6,7,29}$ We fit the model to the data and estimated the remaining parameters.

Viral dynamics model. We extended our previous model of SARS Co-V-2 dynamics, ${ }^{8}$ to include both the lung and nasal passages. In both compartments ( $i \in[L, U], L$ for lung and $U$ for NASAL), we assume that susceptible cells $\left(S_{i}\right)$ are infected at rate $\beta_{i} V_{i} S_{i}$ by SARS-CoV-2 $\left(V_{i}\right)$. SARS CoV-2-infected cells $\left(I_{i}\right)$ die with density dependent rate $\delta_{i} I_{i} I_{i}^{k_{i}}$, where $k_{i}$ describes by how much the first death rate depends on the infected cell density. ${ }^{30}$ This density dependent term represents a combined death of infected cells due to cytopathic effects of the virus and the killing of infected cells due to early immune responses. SARS CoV2 is produced at a rate $\pi_{i}$ and cleared with rate $\gamma_{\mathrm{i}} \cdot{ }^{24}$ Free virus is exchanged between the lungs and nasal passages at rates $\theta_{L U}$ and $\theta_{U L}$.

We also considered the possibility of the emergence of refractory cells. Due to antiviral actions of cytokines such as interferon, it has been experimentally demonstrated that uninfected lung airway cells may become refractory $\left(R_{i}\right)$ at rate $\rho_{i},{ }^{24}$ and that infected cells may convert directly to refractory cells $\left(R_{i}\right)$ at rate $\phi_{i}$. Refractory cells may lose their refractory state and become susceptible at rate $\zeta_{i} \cdot{ }^{24}$ Since we were 
only interested in the viral dynamics in a short span of $\sim 7$ days (with or without treatment), we ignored the death rate of uninfected and refractory cells in the lung, that are usually long-lived.

We also included the possibility of regeneration of susceptible cells during infection. Innate immune cells eliminate virus but can also induce pulmonary tissue damage or endothelium damage as part of this process. ${ }^{31,32}$ The restoration of the respiratory epithelial barrier after an injury is important and may happens within days after viral clearance, ${ }^{33-35}$ depending on the severity of the infection and the extent of lung involvement. Indeed, the proliferation of epithetical cells and progenitor stem cells (or, distal airway stem cells or DASCs) is critical for barrier repair following an inflammatory insult. Following lung injury, the tissue repair process is promoted by immune cells including innate lymphoid cells (ILC-IIs) and macrophages ${ }^{36}$ Epithelial restoration is initiated locally by proliferating alveolar type II (AT2) cells. ${ }^{37}$ We modeled this restoration by adding a logistic proliferation of susceptible and refractory (but not infected ${ }^{38}$ ) epithetical cells with maximum rate $r_{i}$. All the previous mechanisms are modeled by the following differential equation system:

\section{Nasal compartment:}

$$
\frac{d S_{U}}{d t}=r_{U} S_{U}\left(1-\frac{S_{U}+I_{U}+R_{U}}{N_{U}}\right)-\beta_{U} V_{U} S_{U}-\rho_{U} S_{U}+\zeta_{U} R_{U}
$$

$$
\frac{d I_{U}}{d t}=\beta_{U} V_{U} S_{U}-\delta_{U} I_{U} I_{U}^{k_{U}}-\phi_{U} I_{U}
$$

$$
\frac{d V_{U}}{d t}=\pi_{U} V_{U}-\gamma_{U} V_{U}-\theta_{U L} V_{U}+\theta_{L U} V_{L}
$$

$$
\frac{d R_{U}}{d t}=\rho_{U} S_{L}+\phi_{U} I_{U}+r_{U} R_{U}\left(1-\frac{S_{U}+I_{U}+R_{U}}{N_{U}}\right)-\zeta_{U} R_{U}
$$

\section{Lung compartment (measured with BAL):}

$$
\frac{d S_{L}}{d t}=r_{L} S_{L}\left(1-\frac{S_{L}+I_{L}+R_{L}}{N_{L}}\right)-\beta_{L} V_{L} S_{L}-\rho_{L} S_{L}+\zeta_{U} R_{U}
$$

$\frac{d I_{L}}{d t}=\beta_{L} V_{L} S_{L}-\delta_{L} I_{L} I_{L}^{k_{L}}-\phi_{L} I_{L}$

$$
\frac{d V_{L}}{d t}=\pi_{L} V_{L}-\gamma_{L} V_{L}-\theta_{L U} V_{L}+\theta_{U L} V_{U}
$$




$$
\frac{d R_{L}}{d t}=\rho_{L} S_{L}+\phi_{L} I_{L}+r_{L} R_{L}\left(1-\frac{S_{L}+I_{L}+R_{L}}{N_{L}}\right)-\zeta_{U} R_{L}
$$

Here, $N_{U}$ and $N_{L}$ are the maximum carrying capacity of cells in respective compartments (assumed to be the total number of susceptible cells at time of infection).

Model assumptions about lung lesion formation. Although the formation of lung lesions during viral by virus-induced lesions). $G_{L}=N_{L}-S_{L}-I_{L}-R_{L}$

Taking derivative on both sides, we obtain

Notice that this definition of $G_{L}$ is equivalent to $G_{L}=N_{L}-S_{L}-I_{L}-R_{L}$. Under this assumption, the fraction of the lung covered with dead cells would be: $\frac{G_{L}}{N_{L}}$.

Modeling remdesivir therapy. Here we assumed that RDV inhibits viral production. The effect of treatment on the viral production $\pi_{i}$ is reduced by a factor $\left(1-\frac{A_{3 T}}{A_{3 T}+E C_{50 i}}\right)$, where $E C_{50 i}$ is the in vivo $\mathrm{EC}_{50}$ of the nucleoside GS-441524 in the respective compartment $i$.

Model fitting and selection. To fit different versions of the virus dynamics model to the data we used a non-linear mixed effects approach. ${ }^{40,41}$ Briefly, in this approach observed viral load for animal $k$ at time $j$ is modeled as $\log _{10} y_{k j}=f_{V}\left(t_{k j}, \theta_{k}\right)+\epsilon_{V}$ being $f_{V}$ the solution of model for the virus given the individual parameter vector $\theta_{k}$ and $\epsilon_{V}$ the measurement error. Here, the individual-parameter vector $\theta_{k}$ is drawn from 
a population probability distribution. We estimated population parameters using the Stochastic Approximation Expectation Maximization (SAEM) algorithm and the individual parameters using a Markov Chain Monte-Carlo (MCMC) algorithm based on the estimated population distributions. Both algorithms, SAEM and MCMC, were performed using the software Monolix.

We first fit models to nasal and BAL viral loads from untreated animals assuming absent of cell proliferation and refractory cells. Given the lack of observations for the viral load upslope in BAL we assumed $\beta_{L}=\beta_{U}$ (the heterogeneity in early viral dynamics in two regions can still be captured as it $I_{i}(0)=0$ cells $/ \mu$ and $S_{i}(0)=10^{7}$ cells $/ \mu 1$. We also assumed a virus clearance rate to be the same in both compartments $\gamma_{L}=\gamma_{U}=15 /$ day and that $N_{L}=N_{U}=10^{7}$ cells $/ \mathrm{mL}$. We estimated the remaining parameters depending on each model assumptions. The explored competing models on this staged are listed

\section{in Table 2.}

We next fit models to viral load and lung lesion observations from treated and untreated animals.

Here, we explored different competing models listed in Table 3 and described below. We explored models that included cell proliferation and refractory cells in the lungs, fixing $\rho_{U}=0,{ }^{42} \phi_{U}=0$ and $\zeta_{U}=0$. We explored the possibility that AT2 cells proliferate with maximum rate $r_{L}$ after some delay, ${ }^{33}$ i.e. $r_{L}=0$ if cells emerged from susceptible cells in a nonlinear fashion dependent on the concentration of infected cells:

368 (1) with rate $\rho_{i} S_{i} I_{i}$ and (2) with rate $\frac{\rho_{i} S_{i} I_{i}}{I_{50 i}+I_{i}}$. Notice that for the latter when $I_{50 L} \sim 0$ then $\frac{\rho_{i} S_{i} I_{i}}{I_{50 i}+I_{i}} \sim \rho_{i} S_{i}$. We we fixed $\beta$ to the estimated value of the best model when fitting untreated animals and assumed remdesivir

371 reduces virus production $\pi_{i}$ by a factor $\left(1-\frac{A_{3 T}}{A_{3 T}+E C_{50 i}}\right)$ for each compartment $i$. Since we were estimating 
372 both $E C_{50 i}$ and $\pi_{i}$ together we explored fixing the standard deviation of the random effects of $E C_{50 i}$ to 0.1 ,

3730.2 and 1. Finally, we only estimated the fixed effects of $r_{L}, \tau$ and $v$, when applicable. Here, we also

374 assumed $t=0$ as the time of infection with same initial values and fixed parameters $\gamma_{L}, \gamma_{U}, N_{L}$ and $N_{U}$ as

375 before. We estimated the remaining parameters depending on each model assumptions.

376 To determine the best and most parsimonious model among the instances above, we computed the

$377 \log$-likelihood $(\log L)$ and the Akaike Information Criteria ( $\mathrm{AIC}=-2 \log L+2 m$, where $m$ is the number of

378 parameters estimated). We assumed a model has similar support from the data if the difference between its

379 AIC and the best model (lowest) AIC is less than two. ${ }^{43}$ 
A)

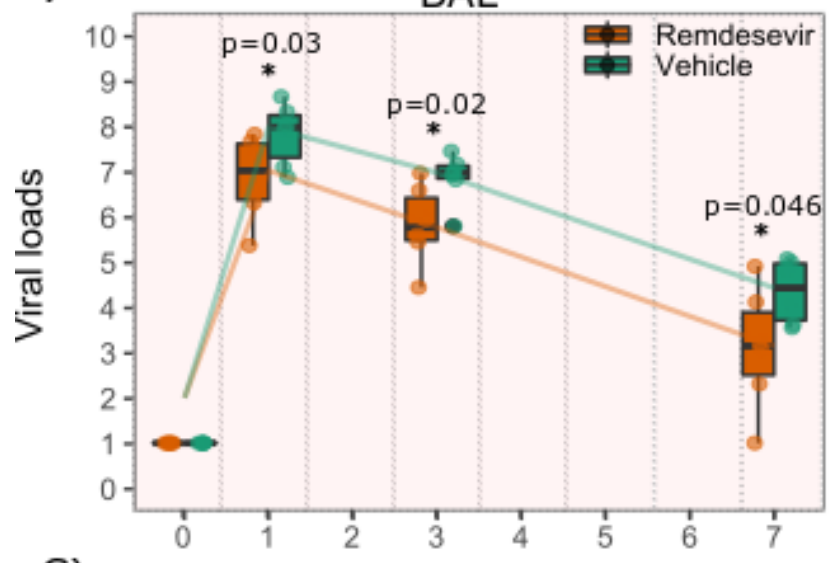

C)

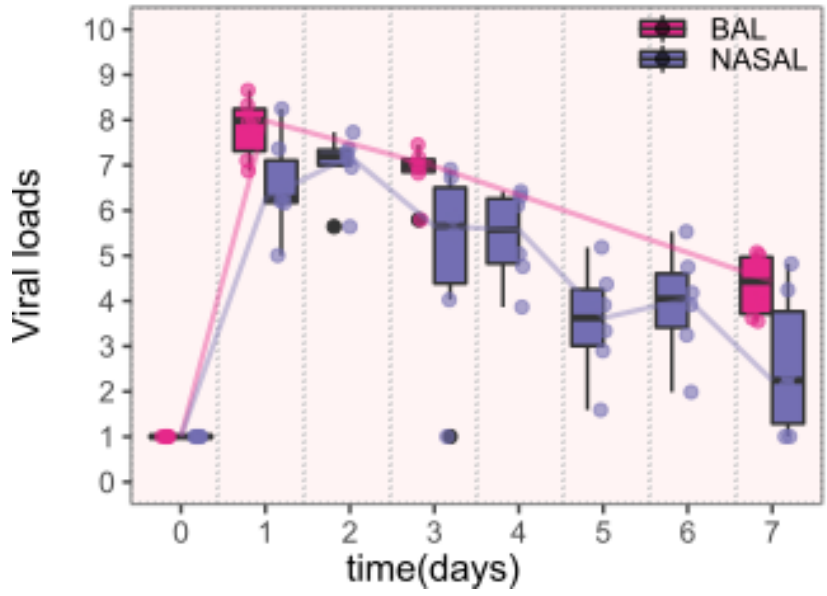

B)

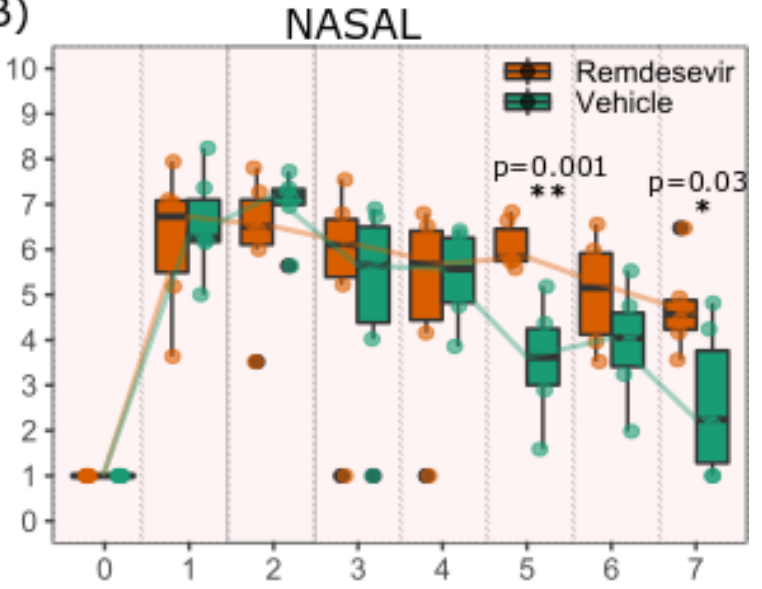

D)

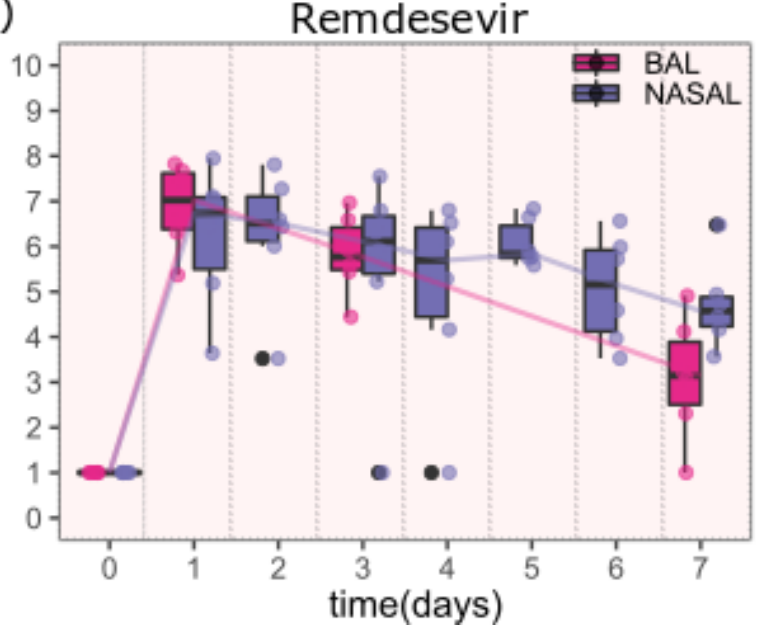

Figure 1: Viral load kinetics following remdesivir treatment in 6 rhesus macaques. A. Decreased

bronchoalveolar lavage (BAL) viral loads in 6 remdesivir treated animals versus 6 vehicle controls at all measured time points. B. Increased nasal viral loads in remdesivir treated animals versus controls at late 


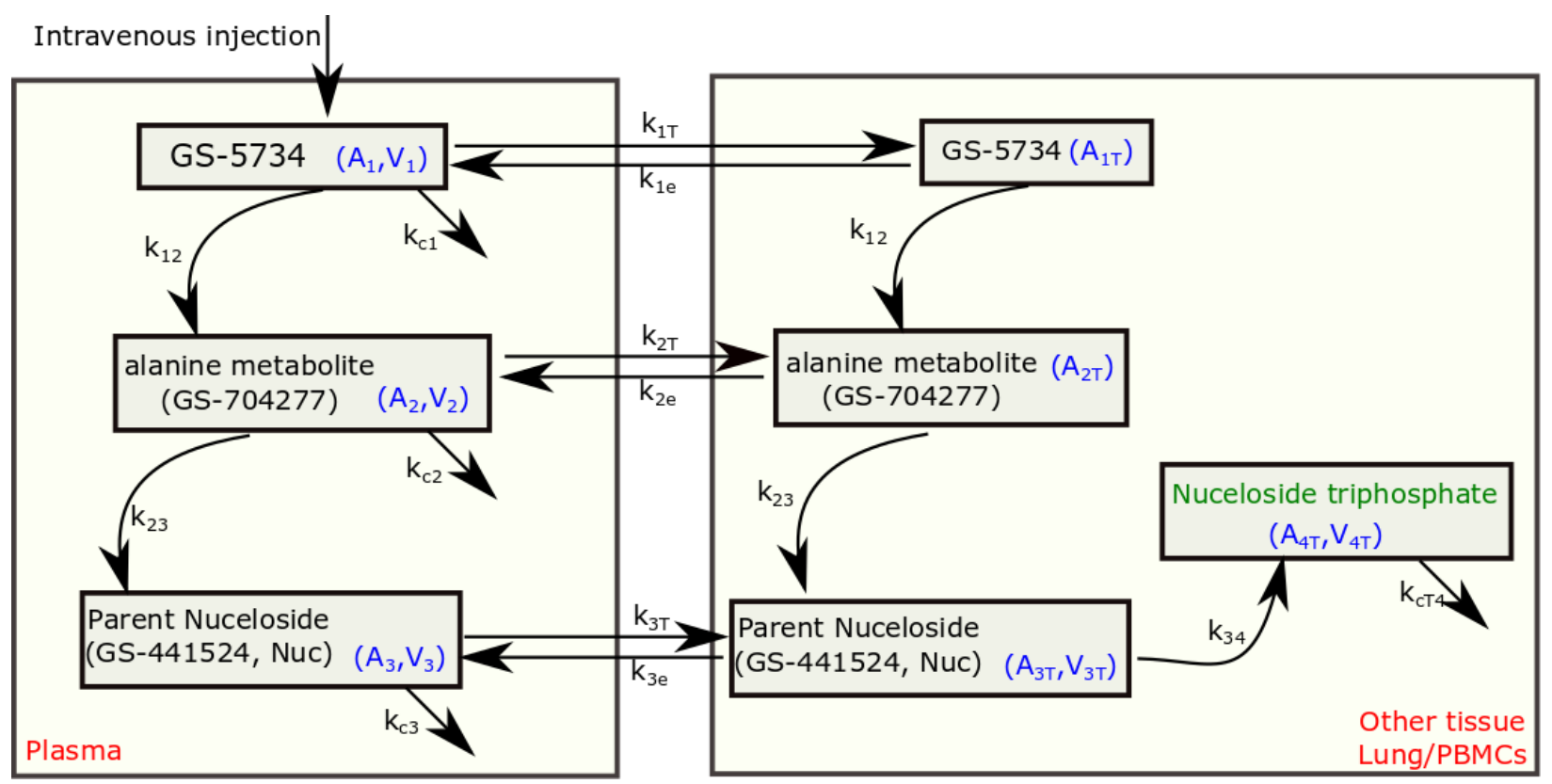

390 Figure 2: Schematic of the remdesivir pharmacokinetic (PK) model. The model includes plasma and

391 tissue levels of remdesivir GS-5734 $\left(A_{1}, A_{1 \mathrm{~T}}\right)$, the alanine metabolite GS-507277 $\left(A_{2}, A_{2 \mathrm{~T}}\right)$ and the parent

392 nucleoside GS-441524 $\left(A_{3}, A_{3 \mathrm{~T}}\right)$ that is phosphorylated in tissue to the active nucleoside triphosphate form

393 of the drug $\left(A_{4 T}\right)$. For remdesivir and the first two metabolites, we modeled the drug distribution from

394 plasma to tissues. 

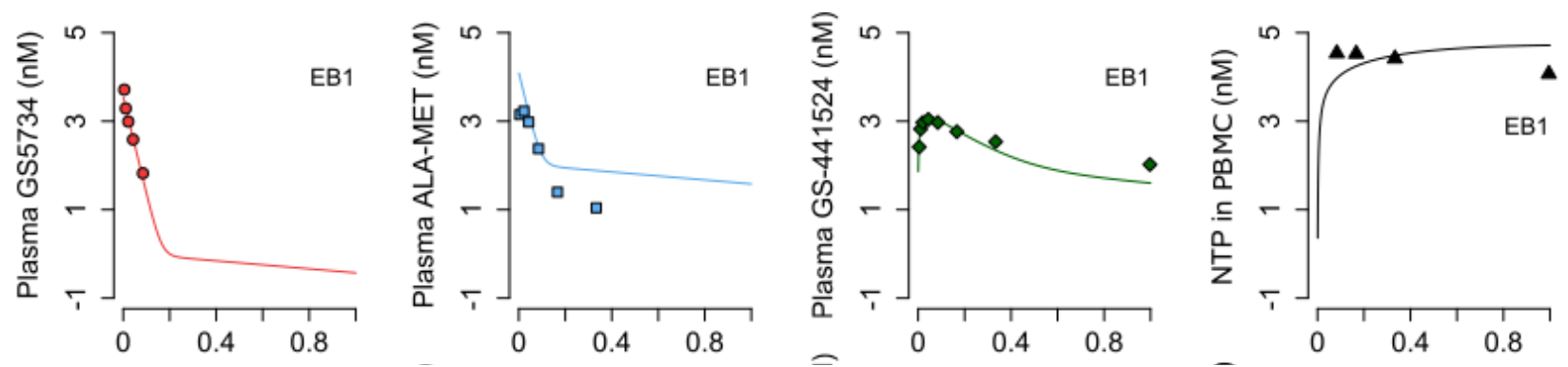

397

\section{B}
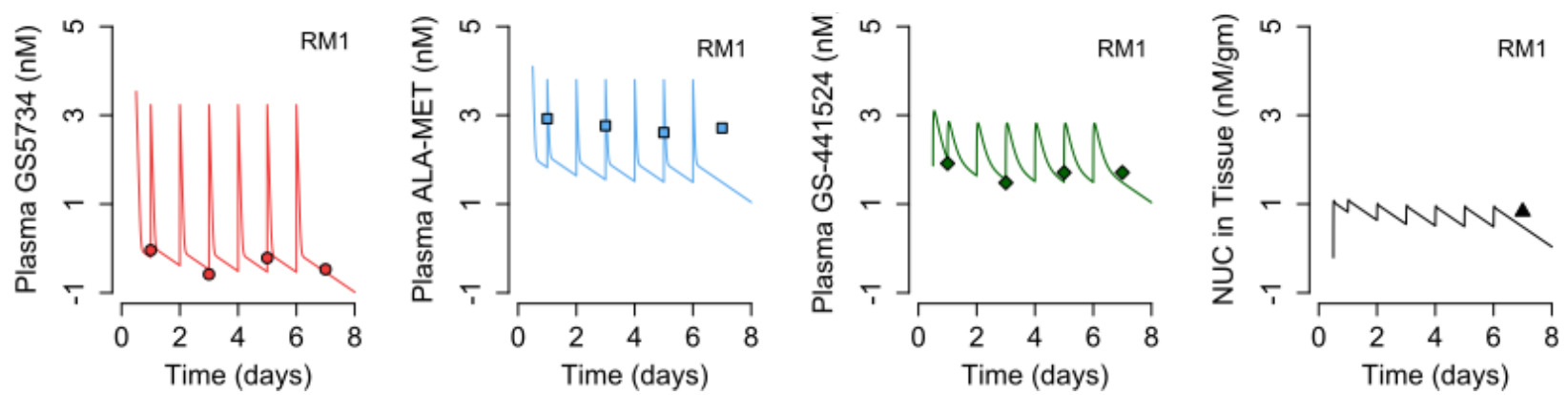

Figure 3: Remdesivir (RDV) pharmacokinetic (PK) model fits to data. A. RDV PK model fits to data animals RM2-RM6 have similar RDV PK profiles. 


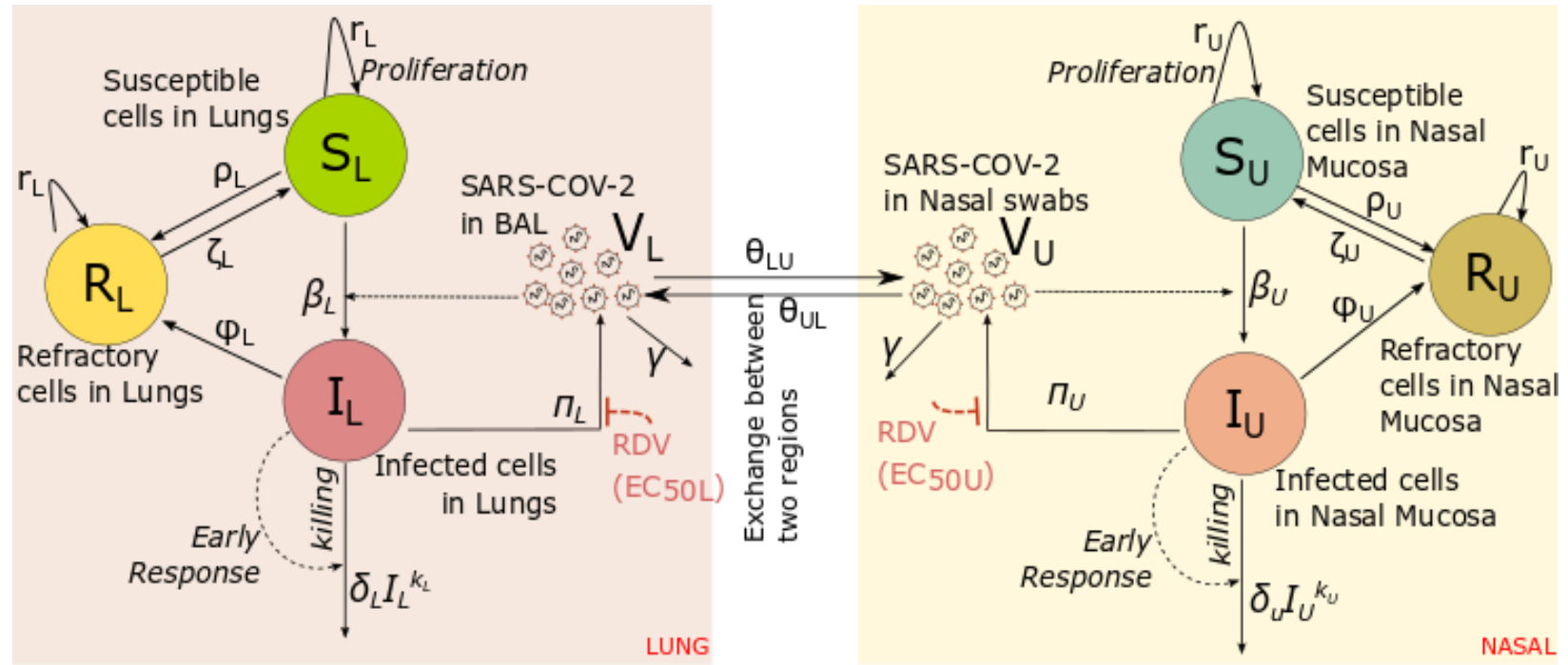

407

B

408

409

410

411

412

413

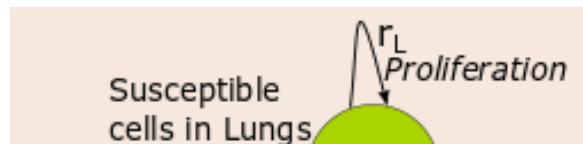

$\mathrm{R}_{\mathrm{L}}$

Refractory

cells in Lungs

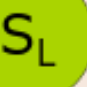

SARS-COV-2

in $\mathrm{BAL}$

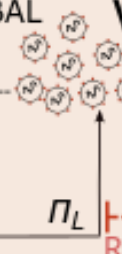

$\Pi_{L}$ s

in Lungs

$$
\text { Response }
$$

LUNG

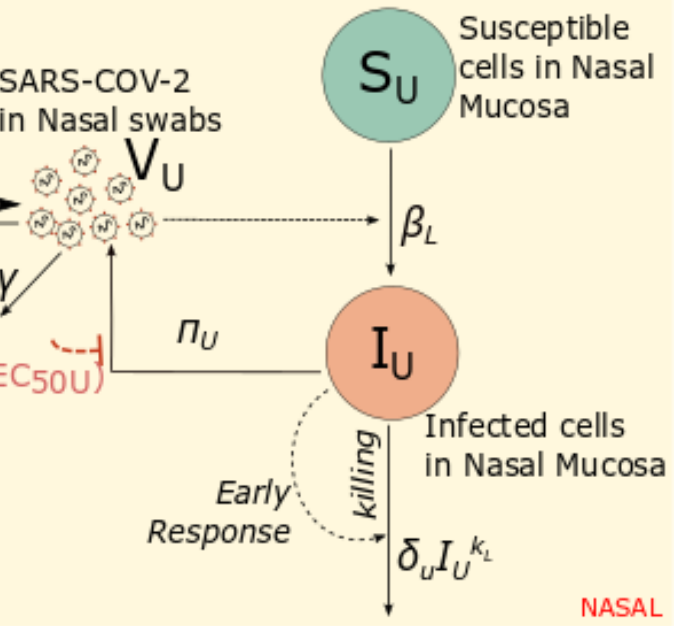

Figure 4: Mathematical models of nasal and lung SARS CoV-2 dynamics and remdesivir therapy. A.

Schematic of a comprehensive viral dynamics model inclusive of all possible compartments and assumptions. B. A reduced model that recapitulates the complete viral load data. Exclusions relative to the complete model include no refractory cell compartment in the nasal passage and no proliferation of susceptible cells in the nasal compartment. 
bioRxiv preprint doi: https://doi.org/10.1101/2020.06.21.163550; this version posted June 22, 2020. The copyright holder for this preprint (which was not certified by peer review) is the author/funder, who has granted bioRxiv a license to display the preprint in perpetuity. It is made available under aCC-BY-NC-ND 4.0 International license.

416

A
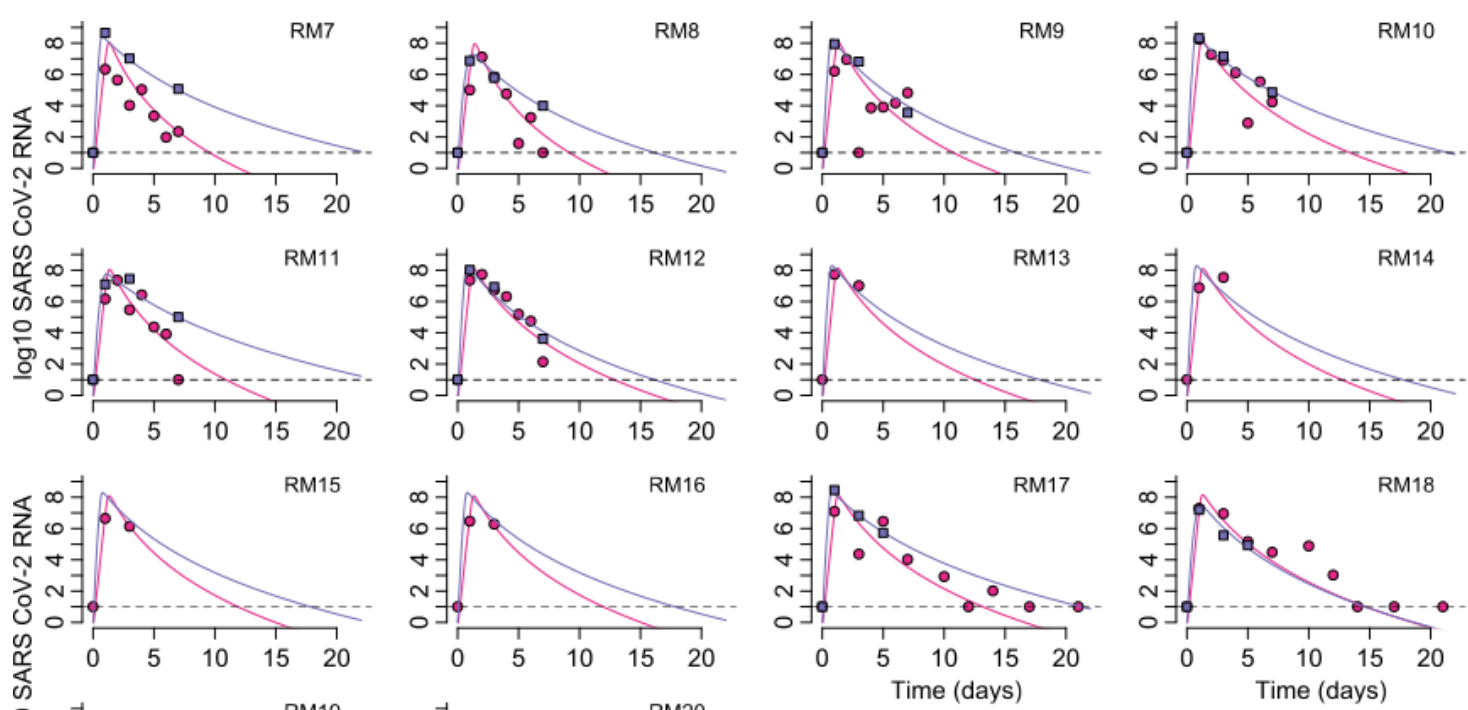

417
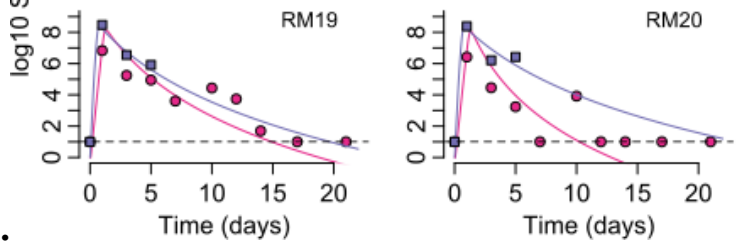

Nasal
BAL
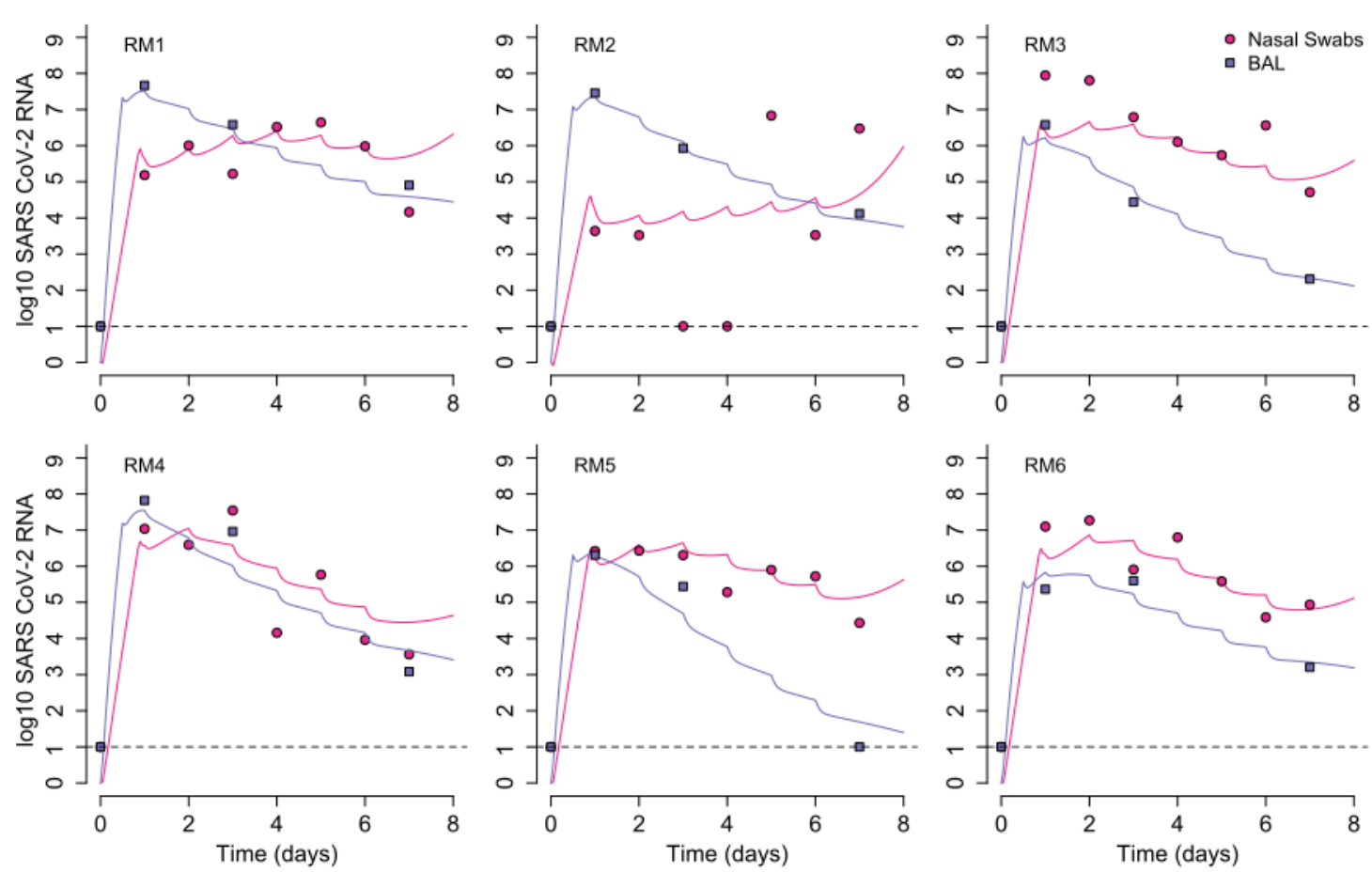

Figure 5: Mathematical model fits to viral load data. A. Fits to untreated animals. RM7-RM12 received a placebo vehicle in direct comparison to the remdesivir treated animals. RM13-20 are from different studies. B. Fits to 6 treated animals who received $10 \mathrm{mg} / \mathrm{kg}$ at day 0.5 and $5 \mathrm{mg} / \mathrm{kg}$ at days 1,2 , 
bioRxiv preprint doi: https://doi.org/10.1101/2020.06.21.163550; this version posted June 22, 2020. The copyright holder for this preprint (which was not certified by peer review) is the author/funder, who has granted bioRxiv a license to display the preprint in perpetuity. It is made available under aCC-BY-NC-ND 4.0 International license.

$4223,4,5$ and 6. Dots (pink=nasal swabs, purple = BAL) are datapoints and lines are model projections. Dots 423 overlying the dotted line are below the limit of detection. Time is in days from infection. 

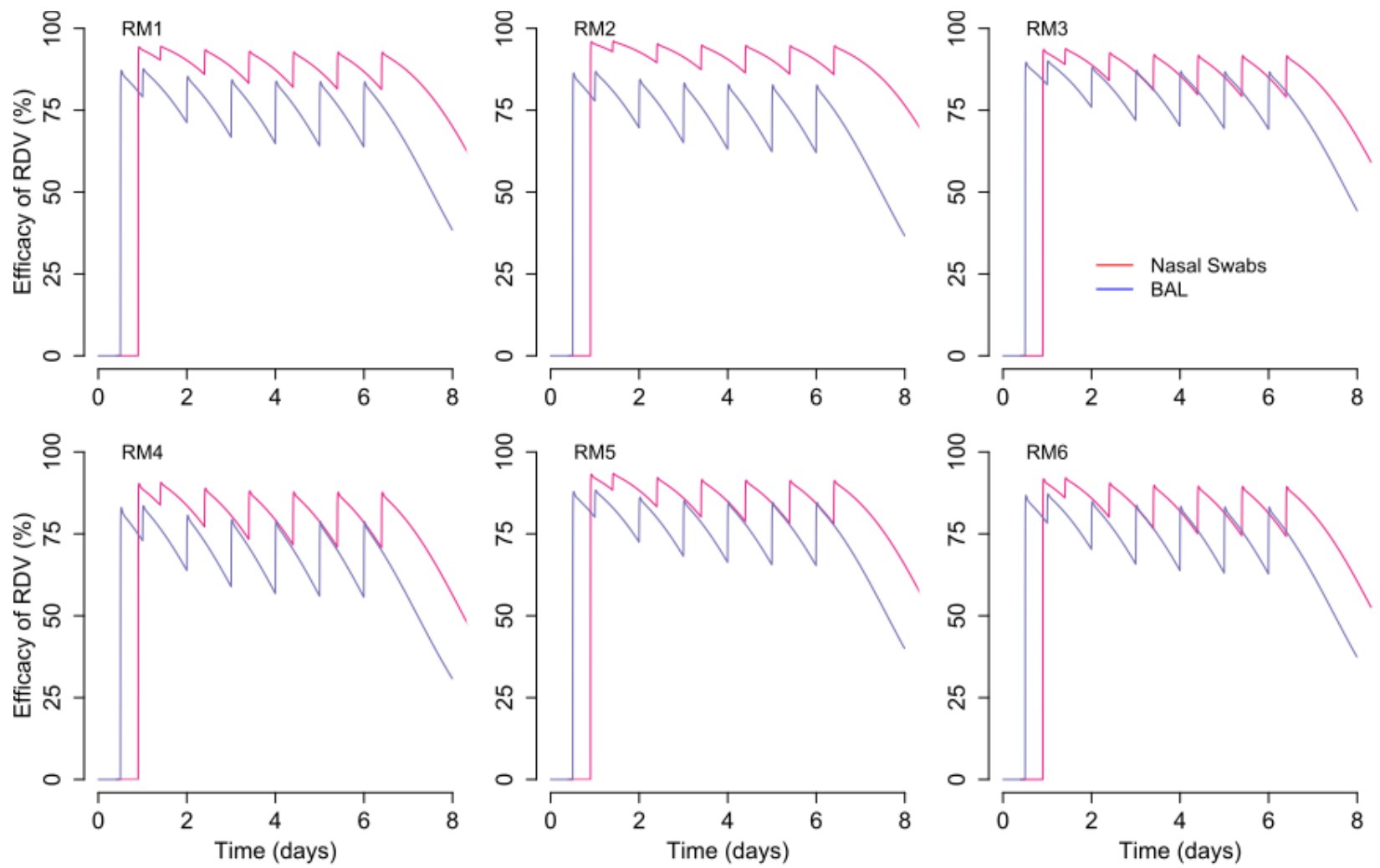

425 Figure 6: Projected direct antiviral efficacy of RDV treatment $\left(\epsilon_{U}\right.$ and $\left.\epsilon_{L}\right)$ in nasal passages (pink

426 line) and the lung (blue line). Over the course of the treatment (from day 0.5 to day 7 ) the projected

427 efficacy of remdesivir in nasal swabs (pink) is higher than in the lung (purple). Projections are based on 428 data from RM1-6. Time is in days from infection. 

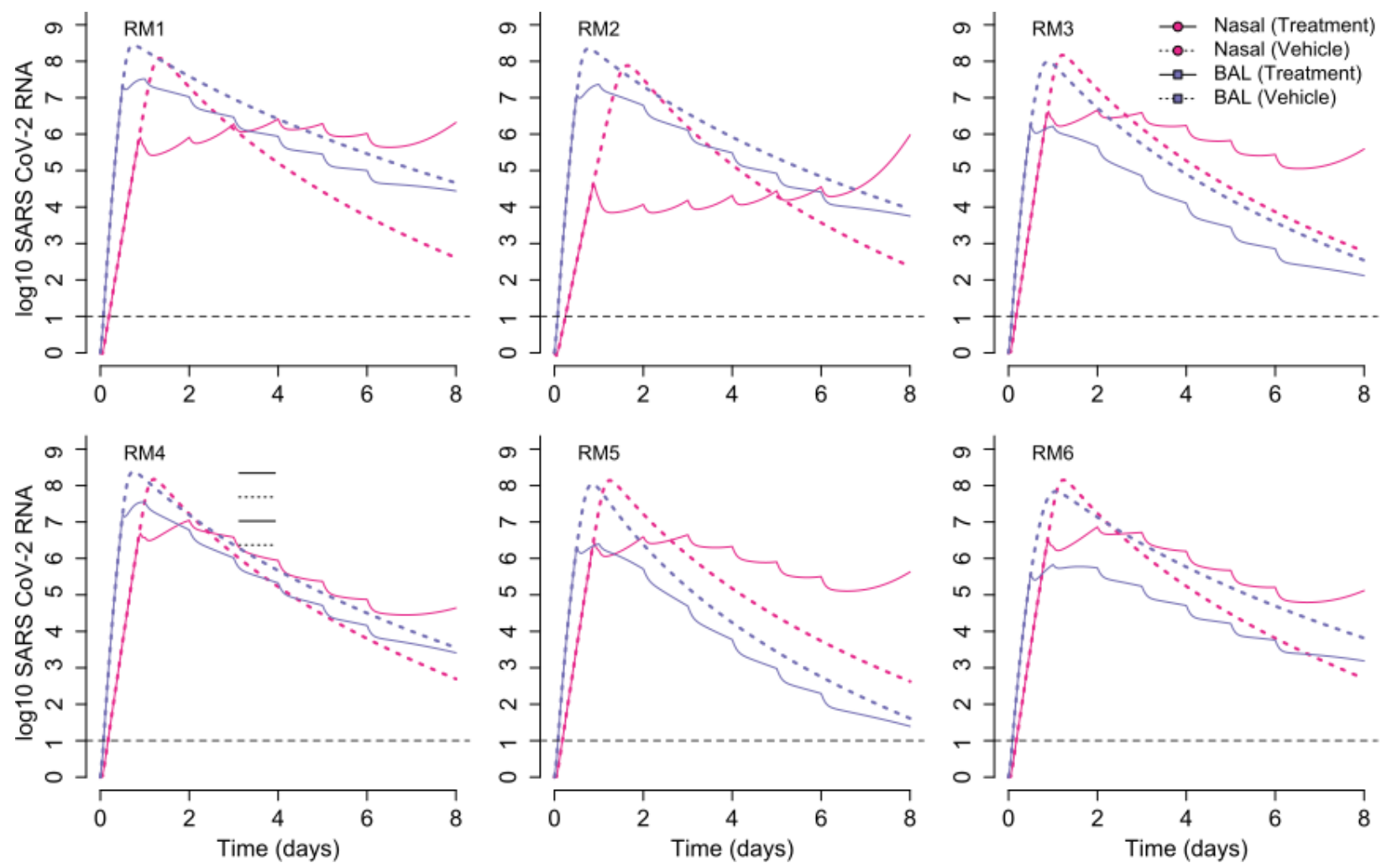

431 lines refer to the simulated viral loads under treatment, and dotted lines are counterfactual simulations

432 assuming no treatment. In the case of the lung (BAL specimens), therapy is projected to lead to consistently

433 lower viral loads. In the case of nasal viral load, therapy temporarily lowers viral load, but viral load is

434 predicted to ultimately persist at higher levels than in the absence of treatment. Simulations are based on 435 data from RM1-6. Time is in days from infection. 

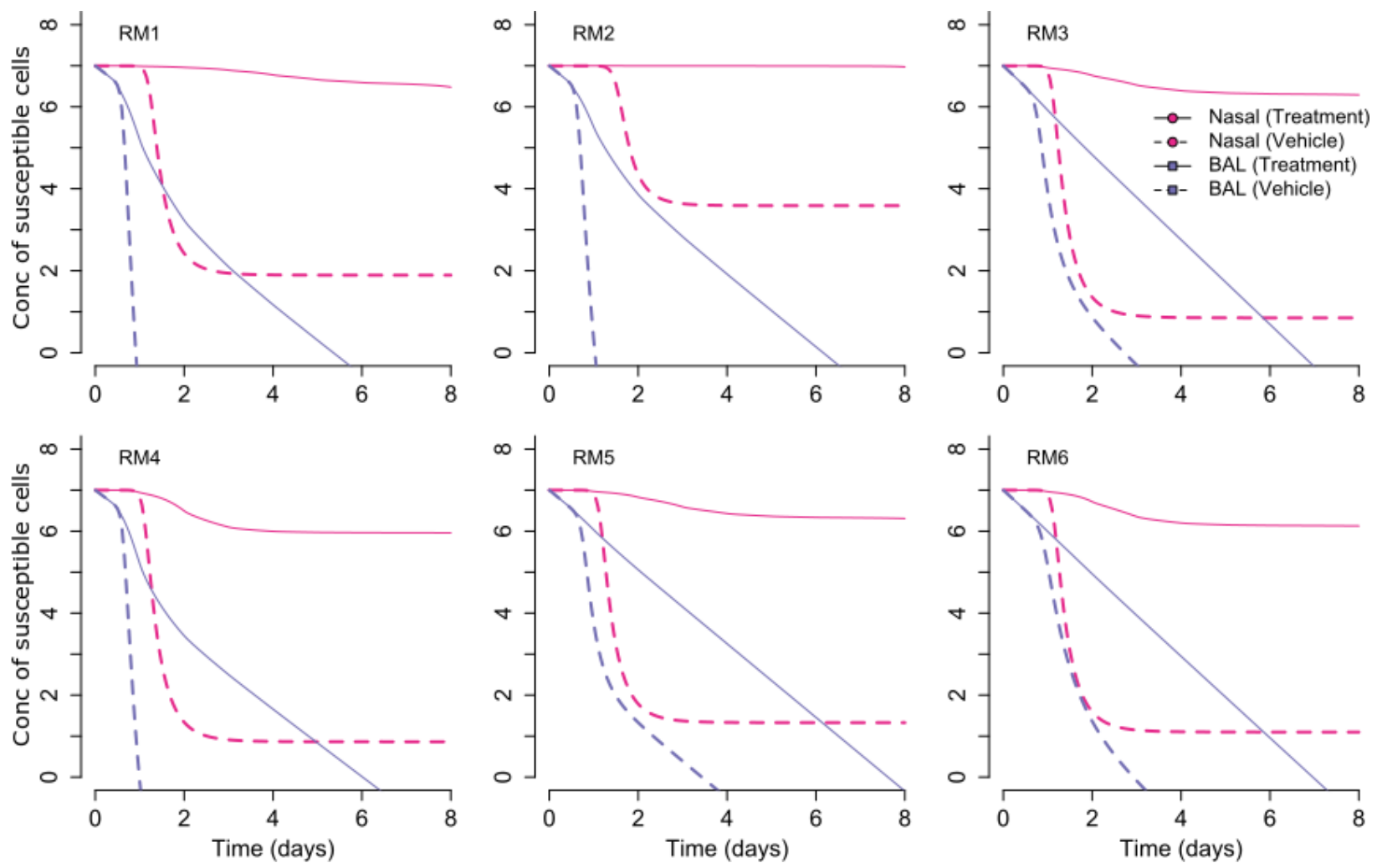

437 Figure 8: Mechanisms of lung protection in remdesivir treated animals. The concentration of

438 susceptible cells is projected for simulations fit to treatment data (solid lines) and counterfactual simulations

439 without therapy (dashed lines). In nasal passages, therapy limits initial depletion of susceptible cells which

440 allows for persistent viral replication rather than elimination. In the lung (BAL specimens), treatment

441 efficacy is lower and susceptible cells can become refractory to infection. The depletion of susceptible cells

442 prevents persistent shedding. Simulations are based on data from RM1-6. Time is in days from infection. 

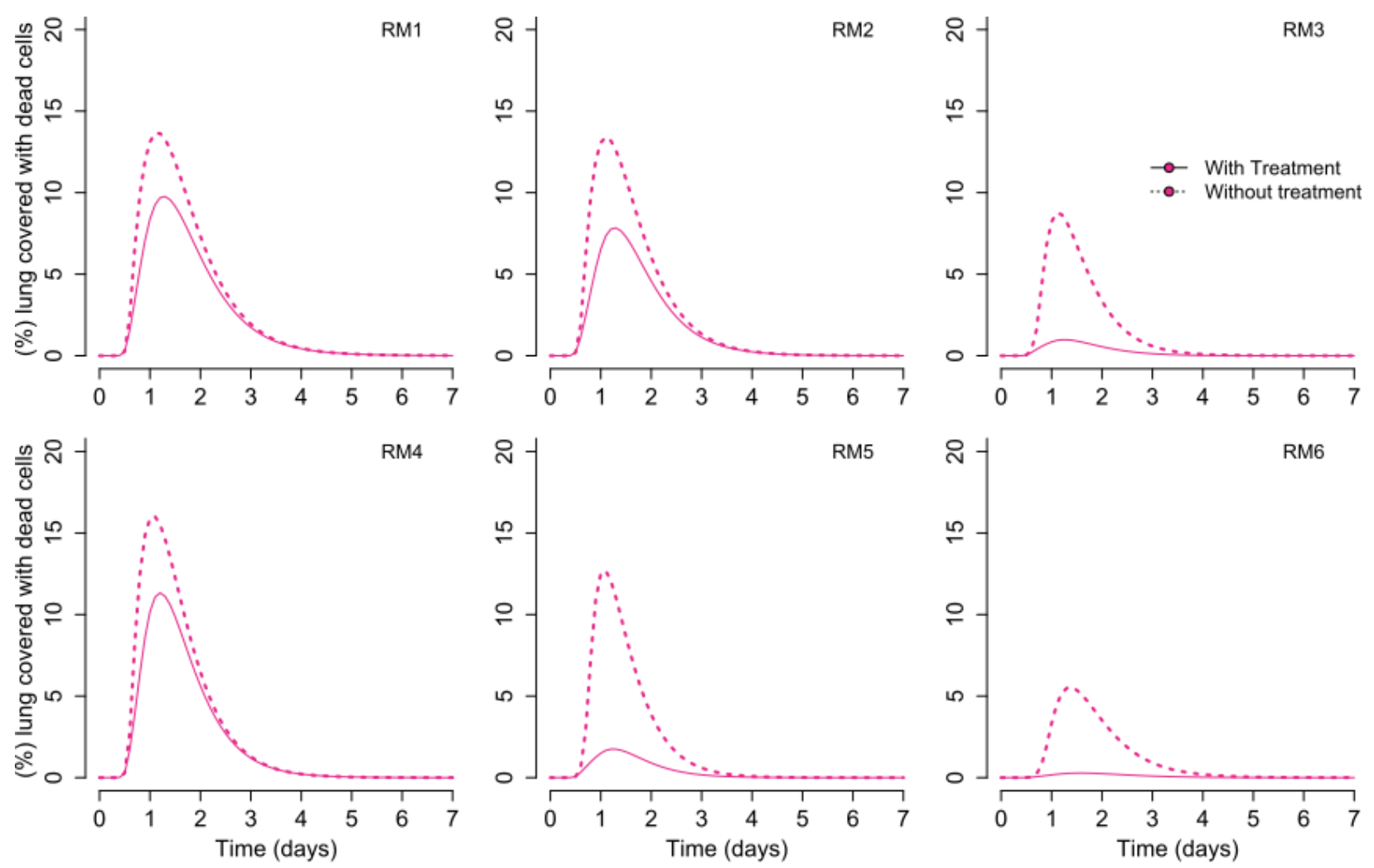

444 Figure 9: Simulated percentage of dead lung target cells as a proxy for lung damage. We define dead

445 cells as the initial total of susceptible cells minus susceptible cells, infected cells and refractory cells in lung

446 at each time point. Treatment lowers the percent of dead cells relative to the counterfactual simulations

447 without therapy. Simulations are based on data from RM1-6. Time is in days from infection. 
A.
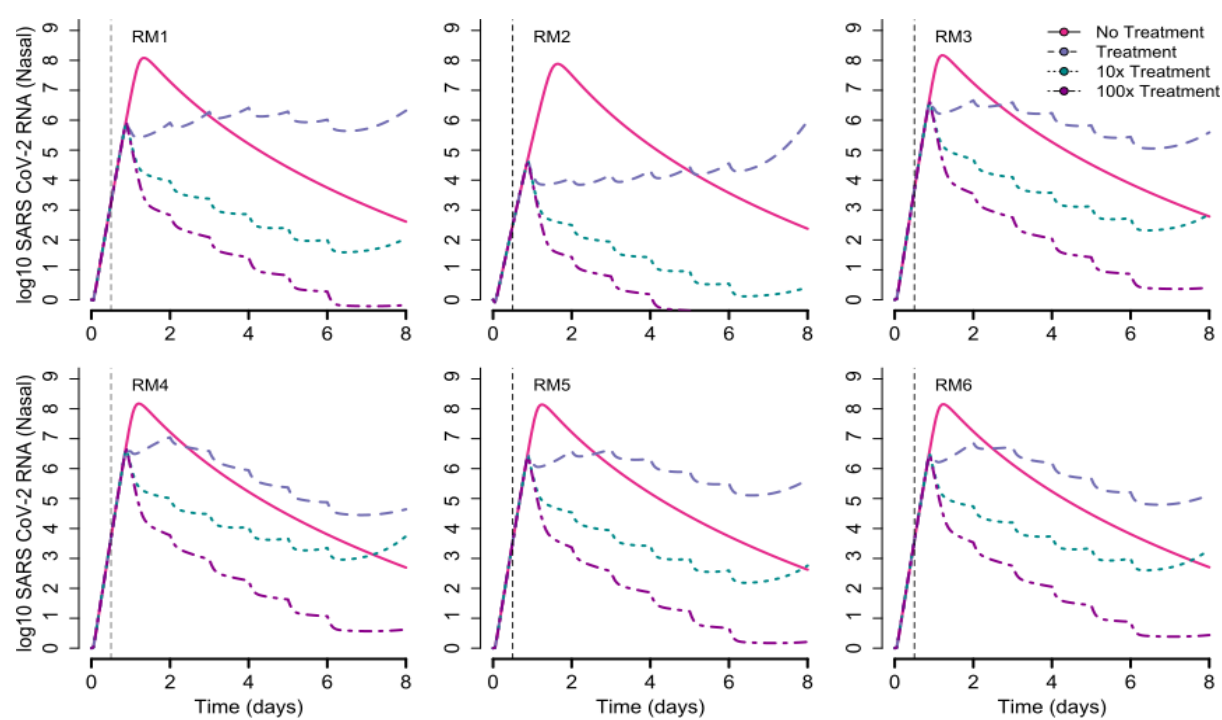

450

B.
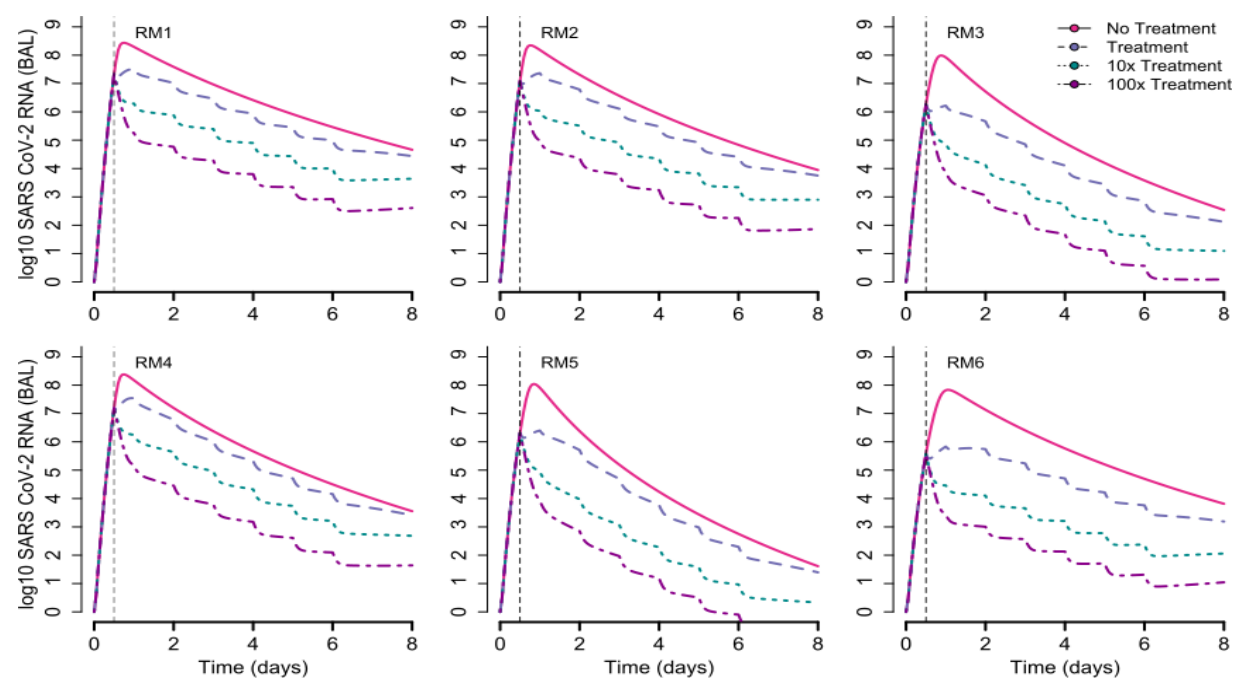

452

Figure 10: Predicted outcome of more potent remdesivir therapy. Therapy is simulated after lowering the in vivo $\mathrm{EC}_{50}$ 10-fold and 100-fold relative to data fitting in Fig $\mathbf{5 b}$. Treatment is started 0.5 days after infection.A. Simulations of nasal viral load. B. Simulations of lung viral loads. Simulations are based on data from RM1-6. 

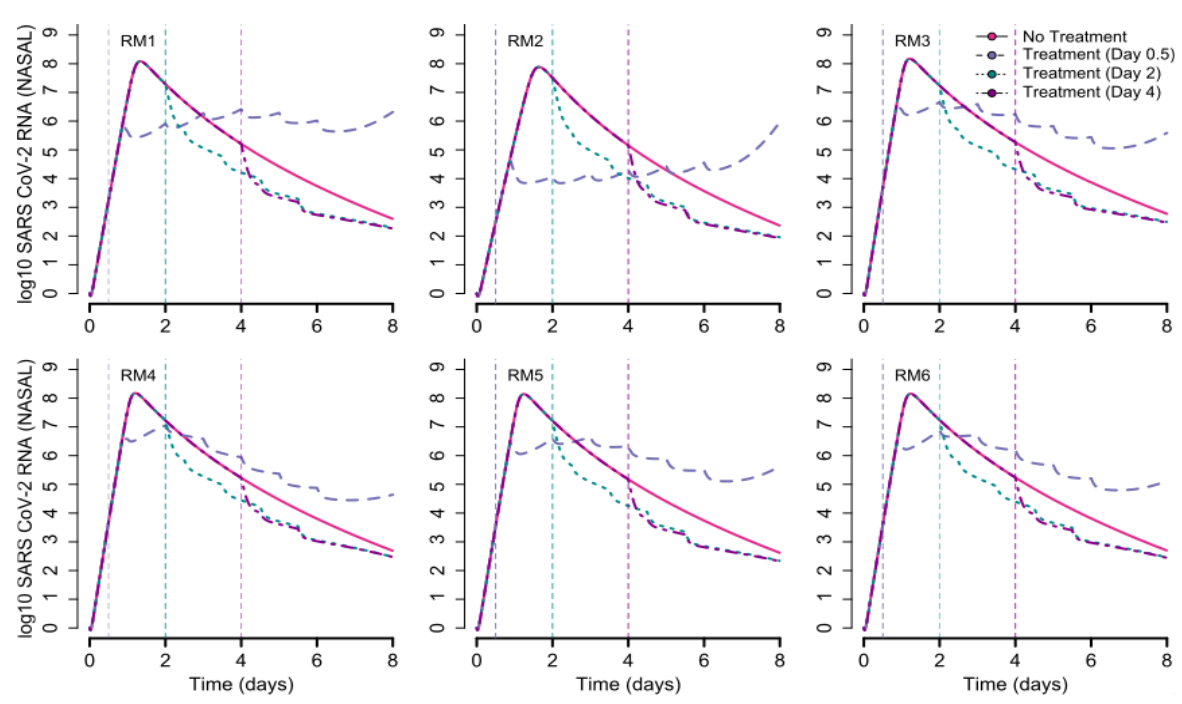

B.
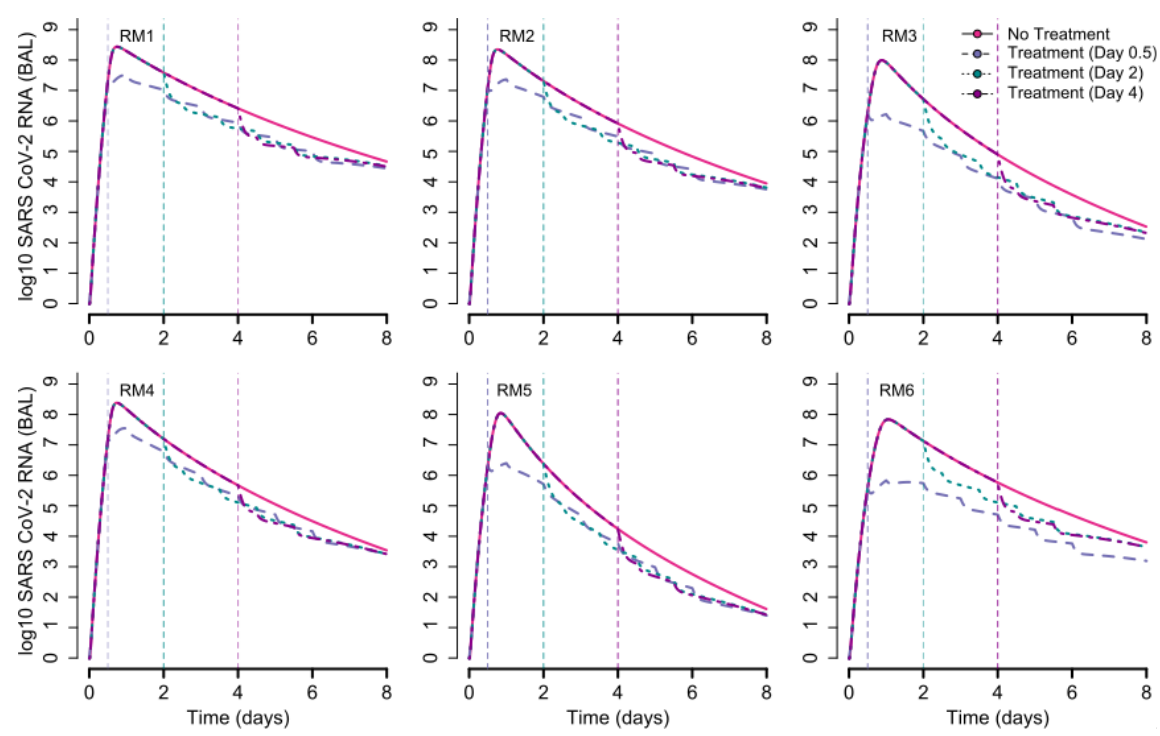

Figure 11: Predicted outcome of later remdesivir therapy. Therapy is simulated with the same antiviral potencies estimated from RM1-RM6 treated at day 0.5 but with initiation at later time points (days 2 and 4). A. Simulations of nasal viral load. B. Simulations of lung viral loads. 
Table 1. Pharmacokinetic model parameters.

\begin{tabular}{|l|c|c|c|c|c|c|c|c|c|c|c|c|c|}
\hline & $k_{1 T}$ & $k_{2 T}$ & $k_{1 e}$ & $k_{2 e}$ & $k_{12}$ & $k_{23}$ & $k_{34 T}$ & $k_{3}$ & $V_{1}$ & $V_{2}$ & $V_{3}$ & $V_{3 T}$ & $V_{4 T}$ \\
\hline RM1 & 33.0 & $\times 10^{3}$ & 0.02 & 12.3 & 1.0 & 989.6 & 158.4 & 7.9 & $10^{-3}$ & $10^{-7}$ & $10^{-5}$ & $10^{-3}$ & $10^{-5}$ \\
\hline RM2 & 33.0 & $\times 10^{3}$ & 0.02 & 12.3 & 1.0 & 989.9 & 168.9 & 7.9 & $10^{-3}$ & $10^{-7}$ & $10^{-5}$ & $10^{-3}$ & $10^{-5}$ \\
\hline RM3 & 33.0 & $\times 10^{3}$ & 0.02 & 12.3 & 1.0 & 989.7 & 162.4 & 7.9 & $10^{-3}$ & $10^{-7}$ & $10^{-5}$ & $10^{-3}$ & $10^{-5}$ \\
\hline RM4 & 33.0 & $\times 10^{3}$ & 0.02 & 12.3 & 1.0 & 990.1 & 173.4 & 7.9 & $10^{-3}$ & $10^{-7}$ & $10^{-5}$ & $10^{-3}$ & $10^{-5}$ \\
\hline RM5 & 33.0 & $\times 10^{3}$ & 0.02 & 12.3 & 1.0 & 990.1 & 166.9 & 7.9 & $10^{-3}$ & $10^{-7}$ & $10^{-5}$ & $10^{-3}$ & $10^{-5}$ \\
\hline RM6 & 33.0 & $\times 10^{3}$ & 0.02 & 12.3 & 1.0 & 989.8 & 175.8 & 7.9 & $10^{-3}$ & $10^{-7}$ & $10^{-5}$ & $10^{-3}$ & $10^{-5}$ \\
\hline
\end{tabular}


469 animals. The model with the lowest Akaike information criteria (AIC) is best supported by the data

470 (denoted in bold). 'Same' implies that the parameter takes the value from the same distribution in two

471 spatial compartments whereas 'different' implies that the parameter has different distributions in two

472 compartments. Here, -2LL represent -2 times the log-likelihood.

\begin{tabular}{|c|c|c|c|c|}
\hline $\boldsymbol{\delta}_{\boldsymbol{i}}$ & $\boldsymbol{k}_{\boldsymbol{i}}$ & $\boldsymbol{\pi}_{\boldsymbol{i}}$ & AIC & $\mathbf{- 2 L L}$ \\
\hline same & same & same & 321.5 & 303.5 \\
\hline different & same & same & 308.1 & 286.1 \\
\hline same & different & same & 326.4 & 304.4 \\
\hline same & same & different & 325.4 & 303.4 \\
\hline different & different & same & 327.3 & 301.3 \\
\hline different & same & different & $\mathbf{3 0 3 . 7}$ & $\mathbf{2 7 7 . 7}$ \\
\hline same & different & different & 317.9 & 291.9 \\
\hline different & different & different & 323.6 & 293.6 \\
\hline
\end{tabular}


475 remdesivir treated animals. The model with the lowest Akaike information criteria (AIC) is best

476 supported by the data (denoted in bold). Models with the inclusion of refractory cells and the proliferation

477 of susceptible cells in lung but not in the nasal passage are better equipped to explain the reduced lung

478 damage in treated animals.

\begin{tabular}{|c|c|c|}
\hline Refractory cells & Proliferation terms & Result \\
\hline $\begin{array}{c}\text { No } \\
\left(\rho_{i} \text { 's }=0 \text { and } \phi_{i}{ }^{\prime} s=0\right)\end{array}$ & $\begin{array}{c}\text { No } \\
\left(r_{i}^{\prime} \mathrm{s}=0\right)\end{array}$ & $\mathrm{AIC}=739.4$ \\
\hline $\begin{array}{c}\text { Yes } \\
\left(\rho_{i}{ }^{\prime} s=0 \text { and } \phi_{i}{ }^{\prime} s \neq 0\right)\end{array}$ & $\begin{array}{c}\text { No } \\
\left(r_{i}^{\prime} s=0\right)\end{array}$ & $\mathrm{AIC}=744.6$ \\
\hline $\begin{array}{c}\text { Yes } \\
\left(\rho_{i}{ }^{\prime} \mathrm{s} \neq 0 \text { and } \phi_{i}{ }^{\prime} \mathrm{s}=0\right)\end{array}$ & $\begin{array}{c}\text { No } \\
\left(r_{i}{ }^{\prime} \mathrm{s}=0\right)\end{array}$ & $\mathrm{AIC}=727.7$ \\
\hline $\begin{array}{c}\text { No } \\
\left(\rho_{i}{ }^{\prime} \mathrm{s}=0 \text { and } \phi_{i}{ }^{\prime} \mathrm{s}=0\right)\end{array}$ & $\begin{array}{c}\text { Yes } \\
\left(r_{U}=0 \text { and } r_{L} \neq 0\right)\end{array}$ & $\mathrm{AIC}=742.7$ \\
\hline $\begin{array}{c}\text { Yes } \\
\left(\rho_{i}{ }^{\prime} s=0 \text { and } \phi_{i}{ }^{\prime} s \neq 0\right)\end{array}$ & $\begin{array}{c}\text { Yes } \\
\left(r_{U}=0 \text { and } r_{L} \neq 0\right)\end{array}$ & $\mathrm{AIC}=730.3$ \\
\hline $\begin{array}{c}\text { Yes } \\
\left(\rho_{i}{ }^{\prime} \mathbf{S} \neq 0 \text { and } \phi_{i}{ }^{\prime} \mathbf{s}=0\right)\end{array}$ & $\begin{array}{c}\text { Yes } \\
\left(r_{U}=0 \text { and } r_{L} \neq 0\right)\end{array}$ & $\mathrm{AIC}=722.4$ \\
\hline
\end{tabular}


Table 4: Estimated parameters under the model depicted in Figure 4b. We also estimated only the

482 fixed effects of three parameters, $\tau=0.42$ days, $v=0.38$ days and $r_{L}=2.0 /$ day while fixing $\beta=1.7 \times 10^{-7}$

483 virions $^{-1} \cdot$ day $^{-1}$.

\begin{tabular}{|c|c|c|c|c|c|c|c|c|c|}
\hline & $k_{U}=k_{L}$ & $\delta_{U}$ & $\begin{array}{c}\log 10 \\
\left(\pi_{L}\right)\end{array}$ & $\delta_{L}$ & $\begin{array}{c}\log 10 \\
\left(\pi_{L}\right)\end{array}$ & $\rho_{L}$ & $\begin{array}{c}E C_{50 U} \\
(\mathrm{nM} / \mathrm{gm})\end{array}$ & $\begin{array}{c}E C_{50 L} \\
(\mathrm{nM} / \mathrm{gm})\end{array}$ & $\begin{array}{c}\text { CATEGOR } \\
\mathrm{Y}\end{array}$ \\
\hline RM1 & 0.09 & 0.91 & 2.5 & 0.51 & 3.2 & 1.9 & 0.8 & 1.9 & RDV \\
\hline RM2 & 0.09 & 0.97 & 2.4 & 0.63 & 3.2 & 2.0 & 0.5 & 1.8 & RDV \\
\hline RM3 & 0.09 & 0.85 & 2.6 & 0.96 & 3.2 & 2.4 & 0.9 & 1.5 & RDV \\
\hline RM4 & 0.09 & 0.89 & 2.6 & 0.73 & 3.2 & 1.9 & 1.0 & 2.0 & RDV \\
\hline RM5 & 0.09 & 0.89 & 2.6 & 1.26 & 3.1 & 2.1 & 0.8 & 1.6 & RDV \\
\hline RM6 & 0.09 & 0.88 & 2.6 & 0.63 & 3.1 & 2.3 & 0.9 & 1.5 & RDV \\
\hline RM7 & 0.09 & 1.14 & 2.5 & 0.51 & 3.2 & 1.9 & NA & NA & VEHICLE \\
\hline RM8 & 0.09 & 1.20 & 2.5 & 0.66 & 3.1 & 2.8 & NA & NA & VEHICLE \\
\hline RM9 & 0.09 & 0.99 & 2.5 & 0.71 & 3.2 & 2.0 & NA & NA & VEHICLE \\
\hline RM10 & 0.09 & 0.80 & 2.6 & 0.53 & 3.2 & 2.0 & NA & NA & VEHICLE \\
\hline RM11 & 0.09 & 0.99 & 2.5 & 0.46 & 3.1 & 2.5 & NA & NA & VEHICLE \\
\hline RM12 & 0.09 & 0.83 & 2.6 & 0.71 & 3.2 & 2.0 & NA & NA & VEHICLE \\
\hline RM13 & 0.09 & 0.85 & 2.6 & 0.64 & 3.2 & 2.0 & NA & NA & VEHICLE \\
\hline RM14 & 0.09 & 0.83 & 2.5 & 0.64 & 3.2 & 2.1 & NA & NA & VEHICLE \\
\hline RM15 & 0.09 & 0.89 & 2.5 & 0.64 & 3.2 & 2.0 & NA & NA & VEHICLE \\
\hline RM16 & 0.09 & 0.89 & 2.5 & 0.64 & 3.2 & 2.0 & NA & NA & VEHICLE \\
\hline RM17 & 0.09 & 0.80 & 2.5 & 0.53 & 3.2 & 2.0 & NA & NA & VEHICLE \\
\hline RM18 & 0.09 & 0.71 & 2.6 & 0.76 & 3.1 & 2.7 & NA & NA & VEHICLE \\
\hline RM19 & 0.09 & 0.70 & 2.5 & 0.58 & 3.2 & 2.0 & NA & NA & VEHICLE \\
\hline RM20 & 0.09 & 1.05 & 2.5 & 0.48 & 3.2 & 2.1 & NA & NA & VEHICLE \\
\hline
\end{tabular}


bioRxiv preprint doi: https://doi.org/10.1101/2020.06.21.163550; this version posted June 22, 2020. The copyright holder for this preprint (which was not certified by peer review) is the author/funder, who has granted bioRxiv a license to display the preprint in perpetuity. It is made available under aCC-BY-NC-ND 4.0 International license.

484

\begin{tabular}{|l|c|c|c|c|c|c|c|c|c|}
\hline & & & & & & & & & \\
\hline Median & 0.09 & 0.89 & 2.6 & 0.68 & 3.2 & 2.0 & 0.8 & 1.7 & RDV \\
\hline Median & 0.09 & 0.87 & 2.5 & 0.64 & 3.2 & 2.0 & NA & NA & VEHICLE \\
\hline
\end{tabular}




\section{References}

1. Schiffer JT, Johnston C, Wald A, Corey L. An early test and treat strategy for SARS-CoV-2. Open Forum Infectious Diseases 2020;doi: 10.1093/ ofid /ofaa232.

2. Wölfel R, Corman VM, Guggemos W, et al. Virological assessment of hospitalized patients with COVID-2019. Nature 2020.

3. Beigel JH, Tomashek KM, Dodd LE, et al. Remdesivir for the Treatment of Covid-19 Preliminary Report. New England Journal of Medicine 2020.

4. Wang Y, Zhang D, Du G, et al. Remdesivir in adults with severe COVID-19: a randomised, doubleblind, placebo-controlled, multicentre trial. Lancet 2020;395:1569-78.

5. Williamson BN, Feldmann F, Schwarz B, et al. Clinical benefit of remdesivir in rhesus macaques infected with SARS-CoV-2. bioRxiv 2020:2020.04.15.043166.

6. WHO R\&D Blueprint - Ad-hoc Expert Consultation on clinical trials for Ebola Therapeutics.

7. Warren TK, Jordan R, Lo MK, et al. Therapeutic efficacy of the small molecule GS-5734 against Ebola virus in rhesus monkeys. Nature 2016;531:381-5.

8. Goyal A, Cardozo-Ojeda EF, Schiffer JT. Potency and timing of antiviral therapy as determinants of duration of SARS CoV-2 shedding and intensity of inflammatory response. medRxiv 2020:2020.04.10.20061325.

9. van Doremalen N, Bushmaker T, Morris DH, et al. Aerosol and Surface Stability of SARS-CoV-2 as Compared with SARS-CoV-1. N Engl J Med 2020.

10. Schiffer JT, Swan DA, Magaret A, et al. Mathematical modeling of herpes simplex virus-2 suppression with pritelivir predicts trial outcomes. Sci Transl Med 2016;8:324ra15.

11. Agostini ML, Andres EL, Sims AC, et al. Coronavirus Susceptibility to the Antiviral Remdesivir (GS-5734) Is Mediated by the Viral Polymerase and the Proofreading Exoribonuclease. mBio 2018;9.

12. Murray JS, Elashoff MR, Iacono-Connors LC, Cvetkovich TA, Struble KA. The use of plasma HIV RNA as a study endpoint in efficacy trials of antiretroviral drugs. AIDS 1999;13:797-804.

13. Siliciano JD, Kajdas J, Finzi D, et al. Long-term follow-up studies confirm the stability of the latent reservoir for HIV-1 in resting CD4+ T cells. Nat Med 2003;9:727-8.

14. Natori Y, Alghamdi A, Tazari M, et al. Use of Viral Load as a Surrogate Marker in Clinical Studies of Cytomegalovirus in Solid Organ Transplantation: A Systematic Review and Meta-analysis. Clin Infect Dis 2018;66:617-31.

15. Chen J, Florian J, Carter W, et al. Earlier sustained virologic response end points for regulatory approval and dose selection of hepatitis C therapies. Gastroenterology 2013;144:1450-5.e2.

16. Ribeiro RM, Perelson AS. Hepatitis B virus viral dynamics: effects of drug dose and baseline alanine aminotransferase. Journal of Hepatology 2002;37:277-9.

17. Schiffer JT, Swan D, Al Sallaq R, et al. Rapid localized spread and immunologic containment define Herpes simplex virus-2 reactivation in the human genital tract. Elife 2013;2:e0288.

18. Schiffer JT, Abu-Raddad L, Mark KE, et al. Mucosal host immune response predicts the severity and duration of herpes simplex virus-2 genital tract shedding episodes. Proc Natl Acad Sci U S A 2010;107:18973-8.

19. Seo S, Xie H, Campbell AP, et al. Parainfluenza virus lower respiratory tract disease after hematopoietic cell transplant: viral detection in the lung predicts outcome. Clin Infect Dis 2014;58:135768 .

20. Histopathologic Changes and SARS-CoV-2 Immunostaining in the Lung of a Patient With COVID-19. Annals of Internal Medicine 2020;172:629-32.

21. Munster VJ, Feldmann F, Williamson BN, et al. Respiratory disease in rhesus macaques inoculated with SARS-CoV-2. Nature 2020.

22. Chemaly RF, Shah DP, Boeckh MJ. Management of respiratory viral infections in hematopoietic cell transplant recipients and patients with hematologic malignancies. Clin Infect Dis 2014;59 Suppl 5:S344-51. 
23. Schiffer JT, Swan DA, Corey L, Wald A. Rapid viral expansion and short drug half-life explain the incomplete effectiveness of current herpes simplex virus 2-directed antiviral agents. Antimicrob Agents Chemother 2013;57:5820-9.

24. Pawelek KA, Huynh GT, Quinlivan M, Cullinane A, Rong L, Perelson AS. Modeling within-host dynamics of influenza virus infection including immune responses. PLoS Comput Biol 2012;8:e1002588.

25. Suthar MS, Zimmerman M, Kauffman R, et al. Rapid generation of neutralizing antibody responses in COVID-19 patients. medRxiv 2020:2020.05.03.20084442.

26. Weiskopf D, Schmitz KS, Raadsen MP, et al. Phenotype of SARS-CoV-2-specific T-cells in COVID-19 patients with acute respiratory distress syndrome. medRxiv 2020:2020.04.11.20062349.

27. Braun J, Loyal L, Frentsch M, et al. Presence of SARS-CoV-2 reactive T cells in COVID-19 patients and healthy donors. medRxiv 2020:2020.04.17.20061440.

28.

https://sidp.org/resources/Documents/COVID19/Matt\%20Remdesivir\%20Handouts\%204.7.2020.

pdf.

29. Sheahan TP, Sims AC, Graham RL, et al. Broad-spectrum antiviral GS-5734 inhibits both epidemic and zoonotic coronaviruses. Sci Transl Med 2017;9.

30. Smith AP, Moquin DJ, Bernhauerova V, Smith AM. Influenza Virus Infection Model With Density Dependence Supports Biphasic Viral Decay. Front Microbiol 2018;9:1554.

31. Newton AH, Cardani A, Braciale TJ. The host immune response in respiratory virus infection: balancing virus clearance and immunopathology. Semin Immunopathol 2016;38:471-82.

32. Gorski SA, Hufford MM, Braciale TJ. Recent insights into pulmonary repair following virusinduced inflammation of the respiratory tract. Curr Opin Virol 2012;2:233-41.

33. Yoo JK, Kim TS, Hufford MM, Braciale TJ. Viral infection of the lung: host response and sequelae. J Allergy Clin Immunol 2013;132:1263-76; quiz 77.

34. Fujino N, Brand OJ, Morgan DJ, et al. Sensing of apoptotic cells through Axl causes lung basal cell proliferation in inflammatory diseases. J Exp Med 2019;216:2184-201.

35. Vaughan AE, Chapman HA. Regenerative activity of the lung after epithelial injury. Biochim Biophys Acta 2013;1832:922-30.

36. Hung LY, Sen D, Oniskey TK, et al. Macrophages promote epithelial proliferation following infectious and non-infectious lung injury through a Trefoil factor 2-dependent mechanism. Mucosal Immunol 2019;12:64-76.

37. Olajuyin AM, Zhang X, Ji HL. Alveolar type 2 progenitor cells for lung injury repair. Cell Death Discov 2019;5:63.

38. Gibbs JD, Ornoff DM, Igo HA, Zeng JY, Imani F. Cell cycle arrest by transforming growth factor beta1 enhances replication of respiratory syncytial virus in lung epithelial cells. J Virol 2009;83:12424-31. 39. Myers MA, Smith AP, Lane LC, et al. Dynamically Linking Influenza Virus Infection with Lung Injury to Predict Disease Severity. bioRxiv 2019:555276.

40. Karlsson M, Janzen DL, Durrieu L, Colman-Lerner A, Kjellsson MC, Cedersund G. Nonlinear mixed-effects modelling for single cell estimation: when, why, and how to use it. BMC Syst Biol 2015;9:52.

41. Chan PL, Jacqmin P, Lavielle M, McFadyen L, Weatherley B. The use of the SAEM algorithm in MONOLIX software for estimation of population pharmacokinetic-pharmacodynamic-viral dynamics parameters of maraviroc in asymptomatic HIV subjects. J Pharmacokinet Pharmacodyn 2011;38:41-61.

42. Baccam P, Beauchemin C, Macken CA, Hayden FG, Perelson AS. Kinetics of influenza A virus infection in humans. J Virol 2006;80:7590-9.

43. Burnham KP, Anderson DR. Model Selection and Multimodel Inference: A Practical InformationTheoretic Approach. 2 ed. New York: Springer-Verlag; 2002. 